This is a postprint version of the following published document:

Rodríguez-Martínez, J. A., Vadillo, G., Fernández-Sáez, J., Molinari, A. (2013). Identification of the critical wavelength responsible for the fragmentation of ductile rings expanding at very high strain rates. Journal of the Mechanics and Physics of Solids, 61, 6, 1357-1376.

DOI: http://dx.doi.org/10.1016/j.jmps.2013.02.003

(C) 2013 Elsevier Ltd.

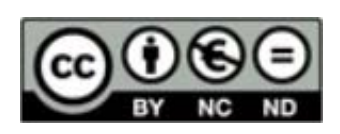

This work is licensed under a Creative Commons Attribution-NonCommercialNoDerivatives 4.0 International License. 


\title{
Identification of the critical wavelength responsible for the fragmentation of ductile rings expanding at very high strain rates
}

\author{
J.A. Rodríguez-Martínez ${ }^{a}$, G. Vadillo ${ }^{a}$, J. Fernández-Sáez ${ }^{a}$, A. Molinari ${ }^{b}$ \\ a Department of Continuum Mechanics and Structural Analysis, University Carlos III of Madrid, Avda. de la Universidad, 30.28911 \\ Leganés, Madrid, Spain

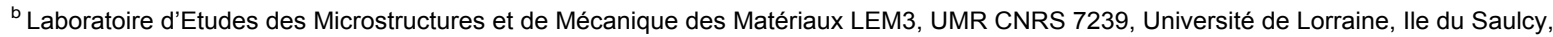 \\ 57045 Metz cedex 1, France \\ ${ }^{\mathrm{n}}$ Corresponding author. Tel. +34 916248460; fax: +34 916249430. E-mail address: jarmarti@ing.uc3m.es (J.A. Rodríguez-Martínez).
}

\begin{abstract}
:
This work examines the mechanisms governing the fragmentation of ductile rings expanding at very high strain rates. Based on previous works three different methodologies have been addressed, namely: fully 3D finite element computations of the radial expansion of ductile rings, numerical simulations of unitary axisymm etric cells with sinusoidal spatial imperfections subjected to tensile loading and a linear perturbation technique derived within a quasi-1D theoretical framework. The results derived from these three different approaches allow for identification of a critical wavelength which dictates the fragmentation of ductile rings expanding at very high strain rates. This critical wavelength is revealed quite independent of the material properties but closely related to the ratio $\left(L_{0} / \phi_{0}\right)^{\text {critical }} \approx 1: 5$ where $L_{0}$ is the fragment size and $\phi_{0}$ is the diameter of the circular section of the ring. This work highlights the fundamental role played by material inertia in the fragmentation at very high strain rates, setting aside the mechanisms associated to the classical statistical theories.
\end{abstract}

\section{Keywords:}

Critical wavelength; Very high strain rates; Fragmentation; Numerical simulations; Stability analysis

\section{Introduction}

Understanding the fragmentation of solids and structures subjected to high velocity impacts or blast loadings has importance in aerospace industry, military applications, civil engineering and geophysical applications. Meteoric cratering, explosive behaviour of projectiles or orbital debris impact on spacecraft structures are examples of situations in which to preserve the integrity of structures subjected to strain rates within the range $>10^{4} \mathrm{~s}^{-1}$ becomes crucial.

From the early studies in this area - dating in the late 18th century - to the present time, an intense debate on the causes which reside behind the fragmentation of solids has been carried out. Particularly, over the last decades large efforts have been made with the aim of describing the inherent or induced flaws causing dynamic failure and fragmentation and its interrelation with the loading conditions.

The characterization of impact fracture and fragmentation emerged as a challenge in experimental mechanics. Niordson (1965) developed a device for the rapid expansion of thin rings to determine materials properties at high strain rates. Compared to the uniaxial impact tensile test (Kàrman and Duwez, 1950; Clark and Wood, 1950) wave disturbances 
are eliminated due to the symmetry of the problem (Fyfe and Rajendran, 1980; Hu and Daehn, 1996; Altynova et al., 1996). The material stretches during loading until homogeneous deformation fails at large strain, leading to flow localization in the form of multiple necking and subsequent fragmentation. From the pioneering work of Niordson (1965), the capability of the expanding rings method to determine fragmentation characteristics of ductile materials has been exploited by several laboratories (Grady and Brenson, 1983; Gourdin, 1989; Altynova et al., 1996; Hu and Daehn, 1996; Juanicotena and Llorca, 1997; Grady and Olsen, 2003; Zhang and Ravi-Chandar, 2006, 2008; Janiszewski and Pichola, 2009; Janiszewski, 2012). Within the typical range of expansion velocities attained in this test - from 50 to $300 \mathrm{~m} / \mathrm{s}$ - the experimental results show that the strain to failure of ductile materials is enhanced by the expansion velocity (Hu and Daehn, 1996; Altynova et al., 1996; Janiszewski, 2012). Moreover, the failure pattern is revealed velocity dependent too, leading to an increasing number of necks and fragments with testing velocity. The experimental evidence revealed that the number of necks formed exceeds the number of fragments. In a recent series of papers, Zhang and Ravi-Chandar (2006, 2008) used a highspeed camera system to obtain high-spatial resolution images showing the ring deformation and fragmentation processes. Through the images recorded it was identified that the onset of fragmentation is caused by the development of a part of the nucleated necks and is completed by extension fracture through the hardened material within these necked regions. The arrested necks result from the arrival of a relieving stress wave from a nearby fracture which removes the driving force before fracture is completed. Thus, from experimentation two fundamental issues arise in the deformation and fragmentation of solids at high strain rates:

1. The physical foundations dictating the increasing ductility with loading rate.

2. The mechanisms governing the necking and fragmentation patterns.

It has been established that increasing ductility in the dynamic regime is controlled by both, inertia and material constitutive behaviour.

- Inertia effect: The role played by material inertia in strain localization has been addressed by Grady (1982) which developed a theoretical model bringing out the stabilizing role played by local inertia effects in fragmentation processes. Meanwhile, Rajendran and Fyfe (1982) glimpsed the stabilizing effect of inertia in the growth of necks represented by geometrical imperfections. Fressengeas and Molinari (1985) developed a stability analysis within the theoretical framework of a 1-D model for uni-axial tension. The study clearly differentiated static and dynamic cases, highlighting the stabilizing effect of inertia on the material behaviour at high strain rates. Somewhat later, Knoche and Needleman (1993) evaluated the influence of material inertia on failure initiation in the round bar tensile test using finite element simulations. They succeeded pointing out the non-linear relation between inertia and necking strain. Han and Tvergaard (1995) revisited the findings brought by Knoche and Needleman (1993) and confirmed by FE simulations the effect of material inertia delaying necking formation in plane strain tensile test specimens. More recently, it is worth noting a number of theoretical and numerical works that have provided additional verification of the benefits provided by material inertia to delay necking formation (Pandolfi et al., 1999; Sørensen and Freund, 2000; Becker, 2002; Mercier and Molinari, 2003, 2004; Zhou et al., 2006c).

- Constitutive behaviour effect: The role played by the constitutive behaviour of the material may be split into the effect of strain hardening and the effect of rate sensitivity both helping to stabilize material behaviour. This was reported by Gosh (1977) who examined theoretically tensile instability and necking in materials showing strain and strain rate dependences of flow stress. The effect of rate sensitivity retarding tensile localization received further attention in the work of Hutchinson and Neale (1977). In this work a non-linear analysis is developed, emphasizing the strong influence of a small degree of material rate sensitivity on necking formation. Moreover, Fressengeas and Molinari (1985) demonstrated the destabilization of plastic flow by thermal softening, i.e. by decreasing strain hardening. Somewhat later, $\mathrm{Hu}$ and Daehn (1996) conducted numerical simulations of rapid radial expansion of rings for viscoplastic materials. They glimpsed the complexity of the interplay between strain hardening and strain rate hardening and they analysed the combined effect of strain and strain rate on necking retardation. In recent years, a number of papers has provided further understanding of the interplay between material behaviour and necking formation (Mercier and Molinari, 2004; Zhou et al., 2006c; Rusinek and Zaera, 2007; Xue et al., 2008; Mercier et al., 2010; Vadillo et al., 2012).

The explanation of the necking and fragmentation patterns experimentally observed has been addressed basically by two different methodologies: statistical analyses and stability analyses.

- Statistical analyses: The starting point of the statistical analyses is the seminal work of Mott (1947) who attempted to capture the characteristic circumferential spacing of fractures - and their statistical distribution in spacing - in metallic casing subjected to rapid expansion. Mott postulated that fragmentation proceeds through the random spatial and temporal occurrence of fractures resulting in a distribution in fragment lengths. Thus, Mott attributed the distribution of fragment sizes to statistical variability in the strain to failure of the material and obtained this distribution through an estimate of the propagation of the unloading or release waves from each fracture event. Further improvements and extensions to the original developments of Mott have been conducted by Grady and co-workers in successive works 
(Grady, 1981; Kipp and Grady, 1985; Grady and Olsen, 2003). The analyses reinforced the idea of the statistical character of the fragmentation process for velocities ranging from 50 to $300 \mathrm{~m} / \mathrm{s}$. They captured the fragment size decrease with increasing loading rate and mass density observed in experiments. The analysis also reflected the increasing fragment size with fracture energy which allows to provide explicit expression for the time to fracture and the fracture strain. Interestingly, the statistical theories have also found application in predicting the fragmentation of brittle solids. The outstanding works of Hild et al. (2003) and Zhou et al. (2005, 2006a, 2006b) rely on the developments of Mott and Grady to provide further explanations on the characteristic fragments size distributions found in brittle solids. Similar to the observations reported for ductile materials, the authors stated that as the loading rate increases both the fragments size and the fragment statistical scatter decrease.

- Stability analyses: The work of Hill and Hutchinson (1975) served as departure for the stability analyses. In this paper they developed a quasi-static bifurcation analysis for a rectangular plate strained under plane strain tension. It was reported that neglecting the role of material inertia short wavelength modes could not be developed and long wavelength modes were favoured. Somewhat later Fressengeas and Molinari (1994), Guduru and Freund (2002), Mercier and Molinari (2003) and Zhou et al. (2006c) showed that the multidimensional character of the stress state in a necked region has a damping effect on short wavelength modes. Moreover, Sørensen and Freund (1998) and Shenoy and Freund (1999) extended the work of Hill and Hutchinson (1975) to dynamic analysis, adding a perturbation of a given wavelength to the fundamental solution. The role of inertia suppressing the long wavelength modes was revealed. This conclusion agreed with previous findings reported by Fressengeas and Molinari (1994) who demonstrated that inertia slows down the growth of long wavelength perturbations. This leads to the selection of a dominant wavelength at each time of the deformation process which dictates the necking spacing. In recent works Mercier and Molinari (2004) and Mercier et al. (2010) applied stability analyses to capture the number of fragments and necks observed experimentally (Altynova et al., 1996; Mercier et al., 2010) in the rapid expansion of rings and hemispheres. Within the higher loading velocities for which there were available data - $\sim 300 \mathrm{~m} / \mathrm{s}$ - the perturbation analyses produced results in rather good agreement with the experimental evidence. However, the difference between theoretical predictions and experiments arises with the impact velocity decrease. Mercier and Molinari (2004) detected that this disagreement obeys to limitations of the stability analyses which do not account for the unloading waves created in the experimental testing which are responsible for slowing down part of the nucleated necks. This suggests that the stability analysis may show better predictive ability as the loading rate increases. At very high strain rates unloading is minimal since the released wave generated by any localization or fracture may not travel far enough quickly to inhibit further nucleation at neighbouring locations (Zhou et al., 2006c). This suggest that if the loading rate is sufficiently high the fragmentation may not be explained by statistical analyses.

Thus, this paper aims to shed light on the mechanisms of fragmentation at very high loading velocities. It is sought to demonstrate the deterministic character of the fragmentation process at sufficiently high strain rates. For that task three different methodologies to describe the radial expansion of ductile rings are applied:

1. Relying on the experimental configuration reported by Grady and Olsen (2003) the radial expansion of ductile rings is simulated using a 3D finite element model. The numerical model has been validated with experimental data and then extended for a range of loading velocities exceeding that available from experimentation. Full 3D computations are a suitable approach to the experimental fragmentation problem. The numerical simulations allow for determining the values of the neck spacing and fragment size upon wide ranges of loading velocities.

2. Relying on the computational model developed by Xue et al. (2008) a circular cross section ring subjected to a radial expansion and with geometrical periodic perturbations is modelled as a unitary axisymmetric cell with sinusoidal imperfection having periodic boundary conditions. A number of computations have been run over a range of cell lengths, loading rates, material behaviours and imperfection amplitudes. Unitary cell computations allow for determining the relationship between necking strain and neck spacing, the latter dictated by the cell length. The cell length with the smaller necking strain defines the configuration of minimum energy dissipation; this is the dominant neck spacing.

3. Relying on the formulation reported by Zhou et al. (2006c) the boundary-value problem of a radially expanding thin ring is formulated. Within a quasi-1D theoretical framework, the equations governing the stretching process of the expanding ring are derived and solved using a linear perturbation method. Stability analyses allow for theoretical determination of the dominant wavelength (wavelength with the fastest grow rate) to be compared with the neck spacing obtained from previous approaches.

Confrontation of results obtained from these three independent methodologies seeks to obtain a unified vision of multiple necking phenomenon.

The paper is organized as follows. In Section 2 the fully 3D finite element model is built and the numerical results are shown. In Section 3 the unitary periodic cell model is presented and computed for different loading cases. In Section 4 the stability analysis is developed and the results interpreted for different loading rates. Section 5 compares the results obtained from the three methodologies addressed. The concluding section outlines the main outcomes of this study. 


\section{Three dimensional finite element modelling of the dynamic expansion of ductile rings}

In this section, the radial expansion of ductile rings is simulated following the fully 3D numerical approach reported by Rusinek and Zaera (2007). This configuration has been shown effective in reproducing the fragmentation mechanics of the ring expansion problem.

\subsection{Numerical configuration}

A Lagrangian 3D finite element model of the rapid expansion of ductile rings is built in ABAQUS/Explicit FE code. The loading condition is a radial velocity $V_{0}$ applied in the inner surface of the ring, which remains constant throughout the entire process, Fig. 1, (Rusinek and Zaera, 2007; Vadillo et al., 2012). The initial condition is a radial velocity $V_{0}$ applied to all the nodes of the model. It should be noted that the initial condition is essential in order to avoid spurious propagation of plastic waves through the thickness of the ring resulting from the abrupt motion of the inner face at $t=0$ while the reminder of the ring is initially at rest. Otherwise, for sufficiently intense waves, the generated plastic wave itself could serve as the trigger for inducing a neck (Needleman, 1991; Xue et al., 2008). These initial and boundary conditions guarantee the uniaxial tensile state in the specimen during loading. The mesh used shows radial symmetry. The ring has been meshed using hexahedral elements (C3D8R in ABAQUS notation) whose aspect ratio is close to 1:1:1, Fig. 1. The integral viscoelastic approach available in ABAQUS/Explicit (2010) has been used to prevent hourglass deformation modes, the scale factor chosen for all hourglass stiffnesses was equal to one. It has been checked that the hourglass energy tends to zero in all the calculations carried out. Five elements are placed along the thickness and width of the sample. The element size is five times smaller than the smallest neck-free segment registered in the simulations. A mesh convergence study has been performed; the time evolution of different critical output variables, namely stress, strain and necking strain, were compared against a measure of mesh density until the results converged satisfactorily. In the numerical simulations the material is defined as elasto-plastic with isotropic plastic hardening. Following the scheme for Huber-Mises plasticity developed by Zaera and Fernández-Sáez (2006) the material definition is considered, in generalized form, strain, rate and temperature dependent.

\subsection{Comparison between finite element simulations and experiments: the case of U6N}

Numerical simulations are conducted and compared with the fragmentation experiments performed by Grady and Olsen (2003) on rings of uranium-6\%-niobium (U6N). According to Zhou et al. (2006c), the material response is defined by a constitutive model that includes power law strain hardening, power law strain rate hardening and linear thermal softening:

$$
\sigma_{y}=\sigma_{0}\left(1+A \varepsilon^{p}\right)^{n}\left(1+\frac{\dot{\varepsilon}^{p}}{\dot{\varepsilon}_{r}}\right)^{m}(1-B T)
$$

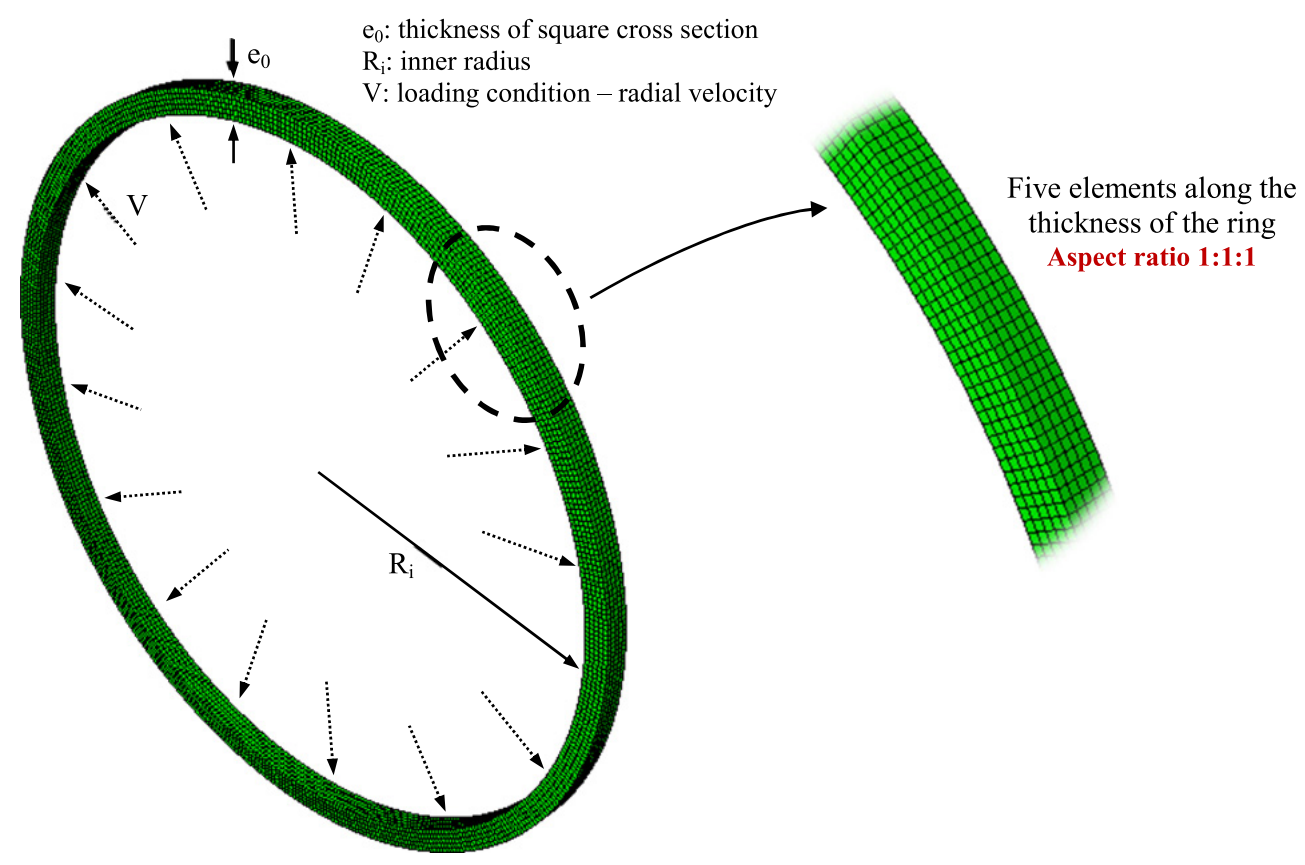

Fig. 1. Finite element model used for the 3D ring expansion simulations. 
It is assumed that the material behaves under adiabatic conditions and that the rate of temperature increase can be calculated as follows:

$$
\rho c_{v} \dot{T}=\beta \sigma \dot{\varepsilon}^{p}
$$

where $\rho$ is the material density, $c_{v}$ is the specific heat and $\beta$ is the Quinney-Taylor coefficient. The parameter values characterizing the behaviour of material U6N are listed in Table 1.

The dimensions of the ring are $R_{i}=15 \mathrm{~mm}$ in inner radius and $e_{0}=0.75 \mathrm{~mm}$ in thickness of square cross section, Fig. 1 . At this point, no geometrical or material imperfections were introduced into the model (Rusinek and Zaera, 2007). The localization is triggered by the numerical round-off which is sufficient to perturb the stress and strain fields. One should note that, although numerical round-offs are essential to trigger flow localization, the nature of the necking pattern is deterministic as recently discussed by Rodríguez-Martínez et al. (2013).

In order to describe the fragmentation of the ring, a failure criterion must be considered. In the computations the material failure is defined by a constant value of the equivalent strain, $\varepsilon_{f}=$ constant, which leads to the element removal. This type of failure criterion, previously adopted by Rusinek and Zaera (2007), has been shown effective to capture the main aspects of the ring fragmentation. The condition that must be imposed to the failure strain is to be large enough to not disturb plastic localization and necking inception. If such condition is fulfilled, the number of necks is not affected by the failure strain level (Vela et al., 2011).

Finite element simulations are conducted using three different failure strain values, $\varepsilon_{f}=1.5, \varepsilon_{f}=2.0$ and $\varepsilon_{f}=2.5$. It has been checked that such failure strain levels preclude excessive element distortion. The numerical simulations capture the characteristic increase in the number of fragments with loading velocity, $25 \mathrm{~m} / \mathrm{s}<V_{0}<300 \mathrm{~m} / \mathrm{s}$ (which corresponds to $1667 \mathrm{~s}^{-1}<\dot{\varepsilon}_{0}<20000 \mathrm{~s}^{-1}$ since the initial strain rate is given by $\dot{\varepsilon}_{0}=V_{0} / R_{i}$ ), independently of the failure strain level considered, Fig. 2. The agreement between experiments and computations is rather satisfactory. However, the

Table 1

Parameter values characterizing the behaviour of material U6N, Eqs. (1) and (2), (Zhou et al., 2006c).

\begin{tabular}{lll}
\hline Symbol & Property and units & Value \\
\hline$\sigma_{0}$ & Reference yield stress (MPa), Eq. (1) & 1000 \\
$A$ & Strain hardening modulus, Eq. (1) & 1.16 \\
$n$ & Strain hardening exponent, Eq. (1) & 0.25 \\
$\dot{\varepsilon}_{r}$ & Reference strain rate (s ${ }^{-1}$ ), Eq. (1) & $10^{-6}$ \\
$m$ & Rate sensitivity exponent, Eq. (1) & 0.005 \\
$B$ & Temperature sensitivity modulus $\left(\mathrm{K}^{-1}\right)$, Eq. (1) & 0.000833 \\
$\rho$ & Density (kg/m $\left.{ }^{3}\right)$, Eq. (2) & 17300 \\
$v$ & Young's modulus (GPa) & 174 \\
$c_{v}$ & Poisson's coefficient & 0.3 \\
$\beta$ & Specific heat (J/kg K), Eq. (2) & 117 \\
\hline
\end{tabular}

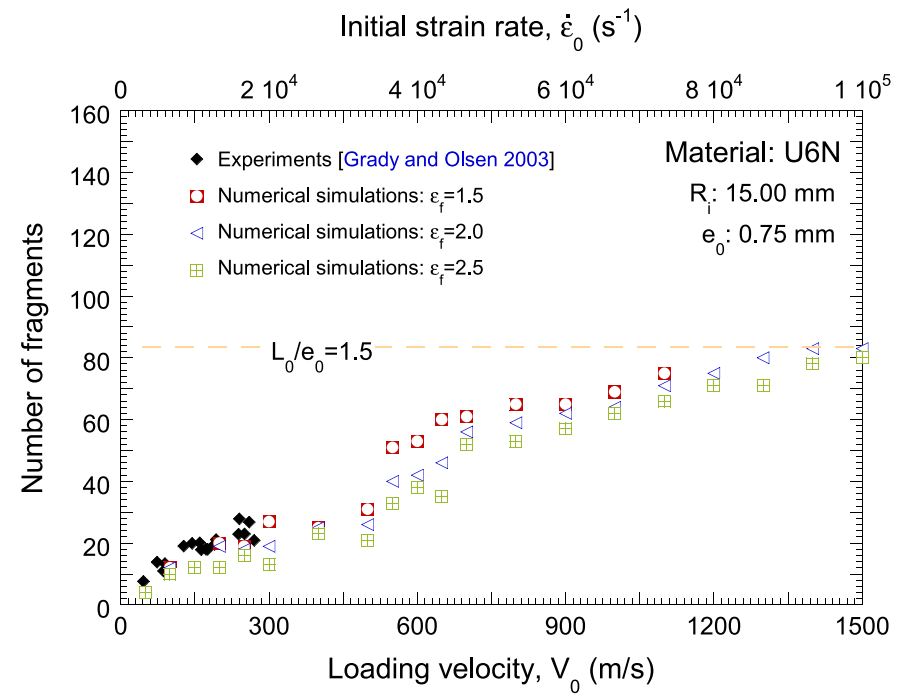

Fig. 2. Number of fragments upon loading velocity $V_{0}$ and initial strain rate $\dot{\varepsilon}_{0}$. Comparison between experiments (Grady and Olsen, 2003) and finite element results for three failure strains, $\varepsilon_{f}=1.5, \varepsilon_{f}=2.0$ and $\varepsilon_{f}=2.5$. Material: U6N. The dashed line refers to the number of fragments at the largest velocity $V_{0}=1500 \mathrm{~m} / \mathrm{s}$. The corresponding mean fragment size (measured in the reference undeformed configuration) is $L_{0}=1.5 e_{0}$ and will be seen to play a crucial role in the following. 
computations have not been limited to the range of loading velocities for which there is experimental data available, but they have been extended for $V_{0}>300 \mathrm{~m} / \mathrm{s}$. Thus, according to Fig. 2, it is possible to establish a classification of the influence of the failure strain level on the numerical simulations attending to the loading velocity:

- Zone I: Comprises the range of loading velocities (may be defined by $V_{0} \lesssim 1000 \mathrm{~m} / \mathrm{s}$ in the present case) for which the failure strain level plays a role on the number of fragments formed. The larger the failure strain the smaller the number of fragments. The number of necks and the number of fragments increases almost linearly with loading velocity, being the number of necks registered considerably larger than the number of fragments, Fig. 3. Such a noticeable difference between number of necks and fragments matches with the experimental evidence and the theoretical considerations reported elsewhere (Mott, 1947; Grady, 1982; Grady et al., 1984; Kipp and Grady, 1985; Grady and Olsen, 2003; Zhang and Ravi-Chandar, 2008; Mercier et al., 2010). The number of necks is determined by recording the strain profile along the perimeter of the ring. Then it is identified the number of excursions from the theoretical material deformation which is given by $\varepsilon^{p}=\ln \left(R(t) / R_{i}\right)$ where $R(t)$ is the time dependent ring radius. During loading, each material location accumulates plastic strain until certain point, then it reaches failure or it is unloaded. Onset of strain localization (onset of necking) is reached at the weakest sections of the ring which are determined by the numerical round-off - i.e. numerical perturbations intrinsic to the FE analysis (Rusinek and Zaera, 2007; Lovinger et al., 2011). Once necking is nucleated at a certain material point, a release wave is generated. This travels down the ring unloading regions encompassed by the release waves. The region that is unloaded by the release wave cannot sustain further plastic strain while other regions continue to experience radial expansion and hence are susceptible to necking development (Zhang and Ravi-Chandar, 2006). The necks nucleated show rather heterogeneous distribution of grow rates. Most necks just develop partially being arrested before reaching material failure as illustrated in Fig. 4. The interplay between necks and failure locations due to unloading waves determines the number of fragments formed. This provides further explanation to the role played by the failure strain level on the numerical simulations. Following Zhang and RaviChandar (2006), a general Weilbull probability density function $f_{w}$ of the form:

$$
\left.f_{w}\left(\left(L_{0} / e_{0}\right)^{n e c k s}\right)=\frac{\kappa}{\lambda} \frac{\left(L_{0} / e_{0}\right)^{n e c k s}-l_{\min }}{\lambda}\right)^{\kappa-1} \exp \left\{-\left(\frac{\left(L_{0} / e_{0}\right)^{n e c k s}-l_{\min }}{\lambda}\right)^{\kappa}\right\}
$$

- was fitted to the measured normalized neck spacing $\left(L_{0} / e_{0}\right)^{\text {necks }}$. In previous expression $\kappa$ is the shape parameter, $\lambda$ is the scale parameter and $l_{\min }$ represents the minimum normalized neck spacing. For example, the histogram of neck spacing displayed in Fig. 5 is related to $V_{0}=100 \mathrm{~m} / \mathrm{s}$ and is fitted by the Weilbull law, Eq. (3). The mean neck spacing is $\left(L_{0} / e_{0}\right)^{\text {necks }} \approx 3.5$ where $L_{0}$ is the neck spacing measured in the reference undeformed configuration.

- Zone II: Comprises the range of loading velocities (may be defined by $V_{0} \gtrsim 1000 \mathrm{~m} / \mathrm{s}$ in the present case) for which the influence of the failure strain value on the number of fragments becomes negligible. The grow rate of the number of fragments and necks considerably decreases upon the loading velocity. At the largest loading velocities considered, the number of fragments and necks practically coincide and their dependence with the loading speed becomes strictly reduced, Fig. 3. The number of fragments predicted tends to saturation, $L_{0} / e_{0} \approx 1.5$. Here, as in Fig. $2, L_{0}=1.5 e_{0}$ represents the value of the mean fragment size at the largest velocities. Within this range of loading velocities, more fractures develop at shorter distances since the unloading generated by any localization may not travel far enough quickly to inhibit further nucleation at neighbouring locations (Zhang and Ravi-Chandar, 2006). The fragmentation

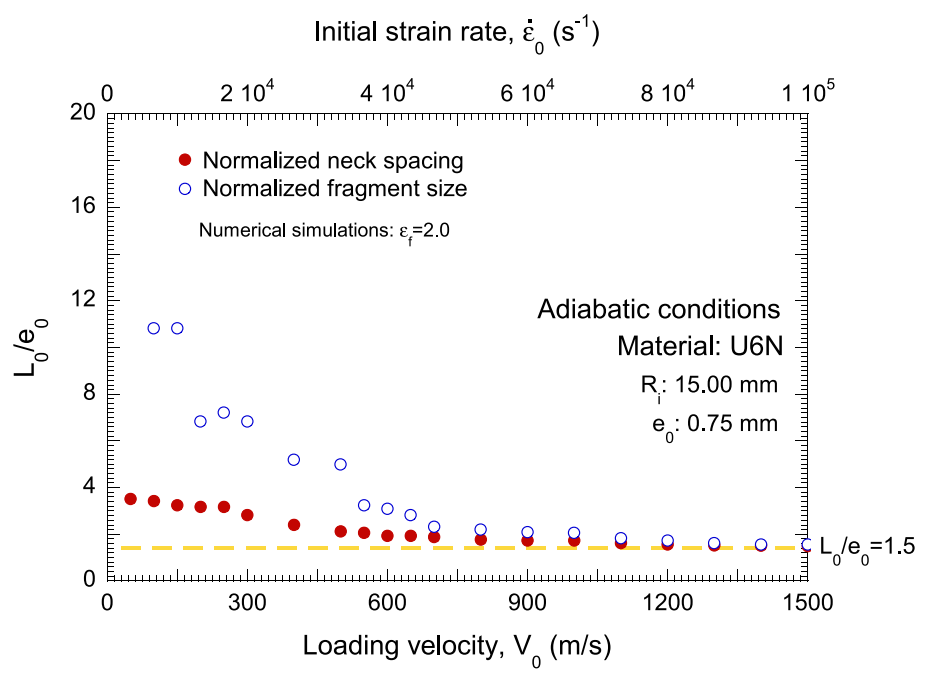

Fig. 3. Finite element results. Normalized mean fragment size and normalized neck spacing upon loading velocity $V_{0}$ and initial strain rate $\dot{\varepsilon}_{0}$. Failure strain $\varepsilon_{f}=2.0$. Material: U6N. $L_{0}$ is measured in the reference undeformed configuration. 


\section{Material: U6N $-V_{0}=\mathbf{2 0 0} \mathbf{m} / \mathrm{s}$}
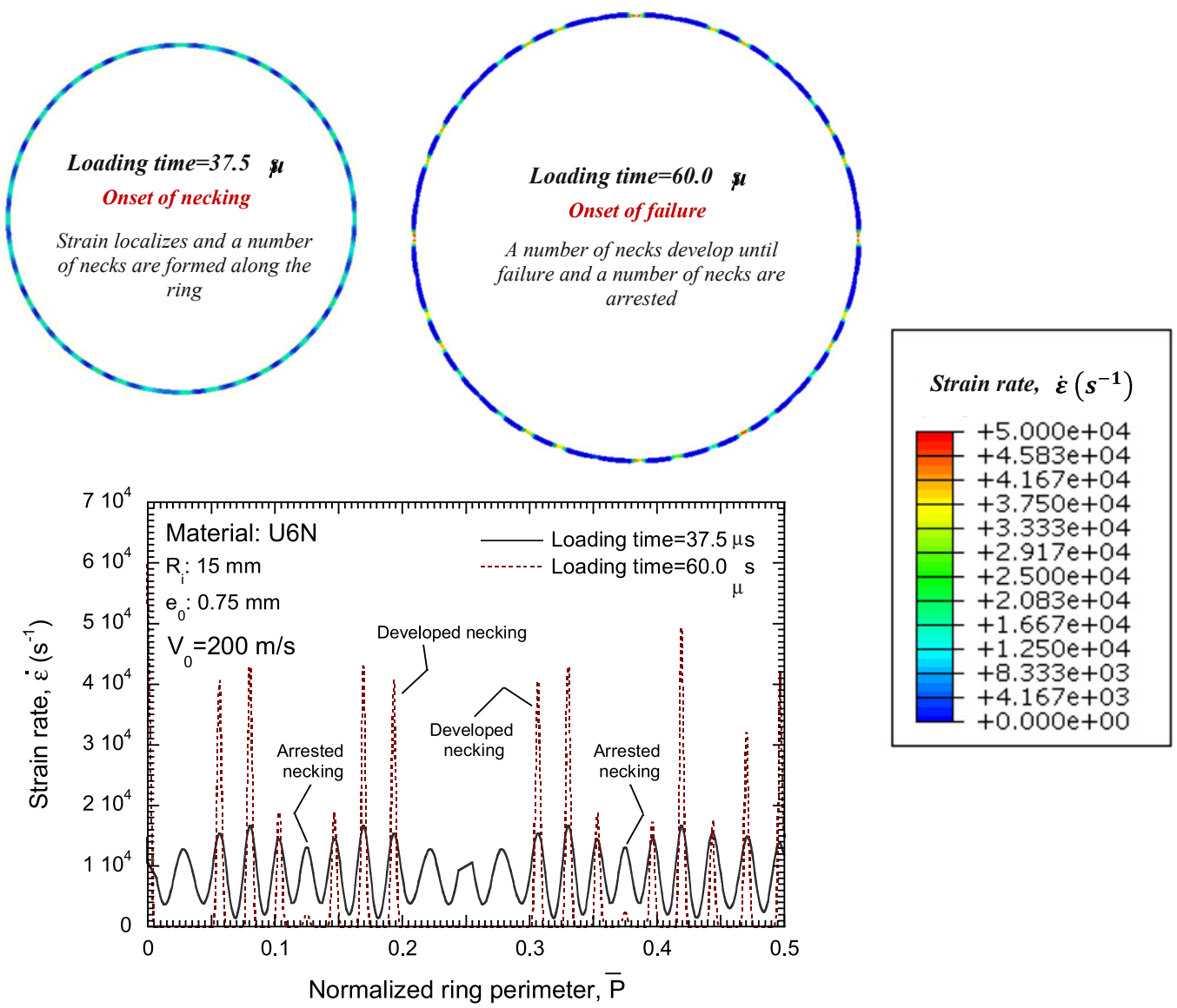

Fig. 4. Finite element results. Strain rate along (half of) the perimeter of the ring at different loading times. $V_{0}=200 \mathrm{~m} / \mathrm{s}$. Failure strain $\varepsilon_{f}=2.0$. Material: U6N.

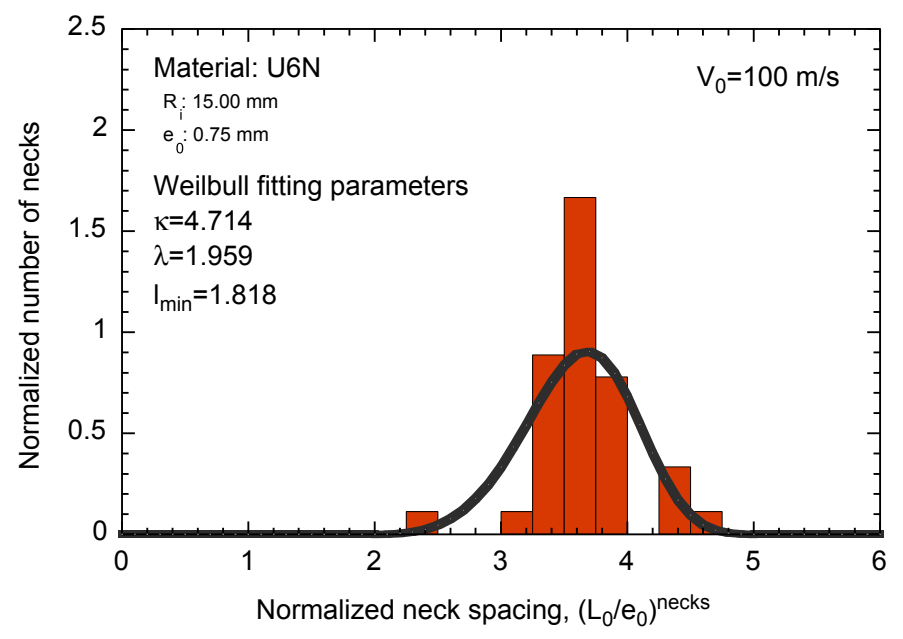

Fig. 5. Finite element results. Normalized number of necks as a function of the normalized neck spacing $\left(L_{0} / e_{0}\right)^{\text {necks }} . V_{0}=100 \mathrm{~m} / \mathrm{s}$. Fitting parameters in Eq. (3). $\kappa=4.714, \lambda=1.959$ and $l_{\min }=1.818$. Material: U6N.

process cannot be considered any more a random spatial and temporal occurrence. Equally spaced (almost) and equally intense (almost) necks are formed (practically) simultaneously all along the ring which makes possible that most necks nucleated develop until failure, Fig. 6. This suggests that the interplay between neighbouring necks is minimal which explains that different values of the failure strain may provide very close numerical results. The Weilbull probability density function, Eq. (3), has been fitted to a high loading velocity simulation, $V_{0}=1600 \mathrm{~m} / \mathrm{s}$, Fig. 7 , being the neck spacing quite homogeneous. In comparison with Fig. 5 the mean neck spacing has been drastically dropped. This is illustrated by the systematic reduction of the scale parameter $\lambda$ with loading velocity (see comparison between Figs. 5 


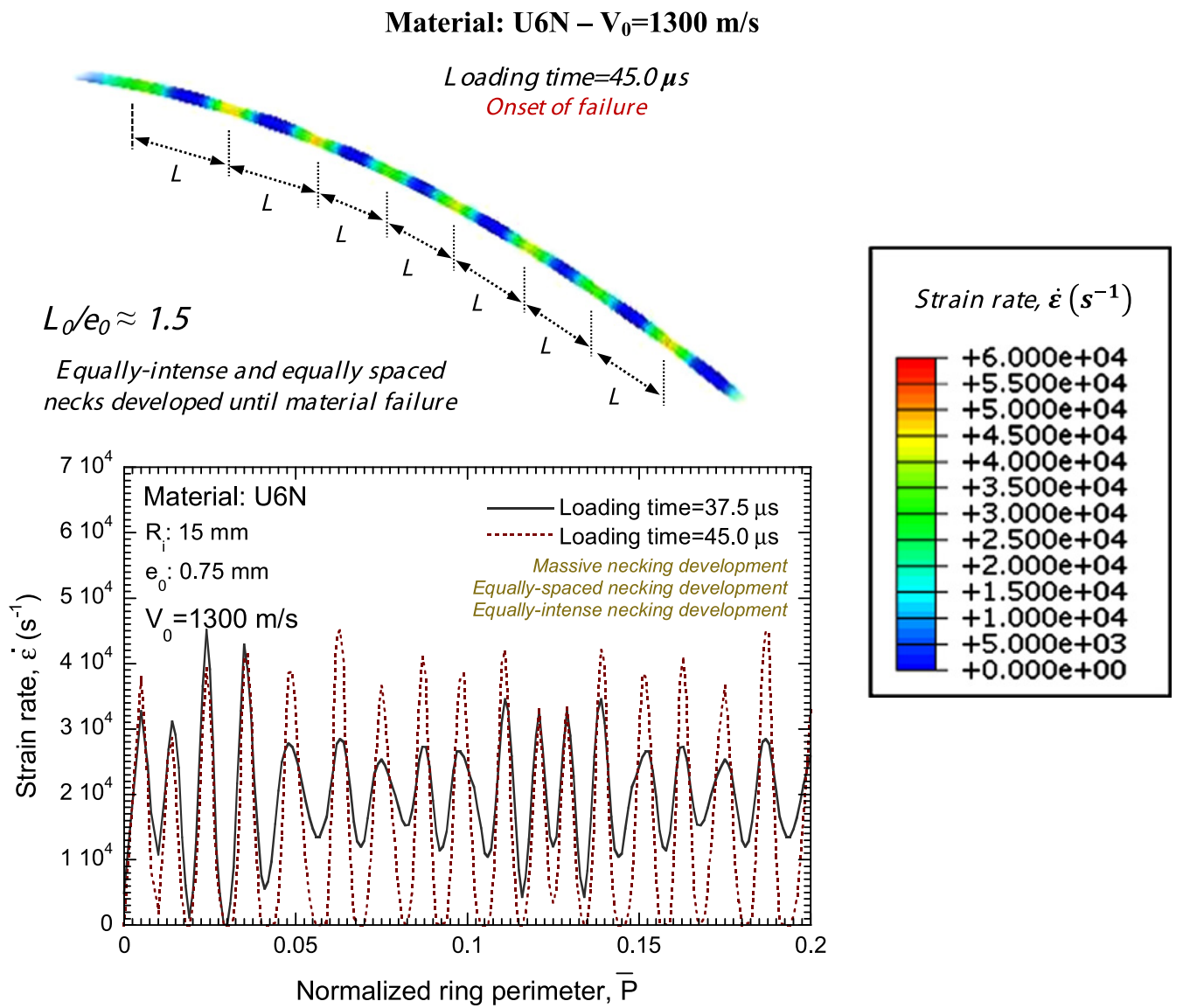

Fig. 6. Finite element results. Strain rate along $(1 / 5$ of $)$ the perimeter of the ring at different loading times. $V_{0}=1300 \mathrm{~m} / \mathrm{s}$. Failure strain $\varepsilon_{f}=2.0$. Material: U6N.

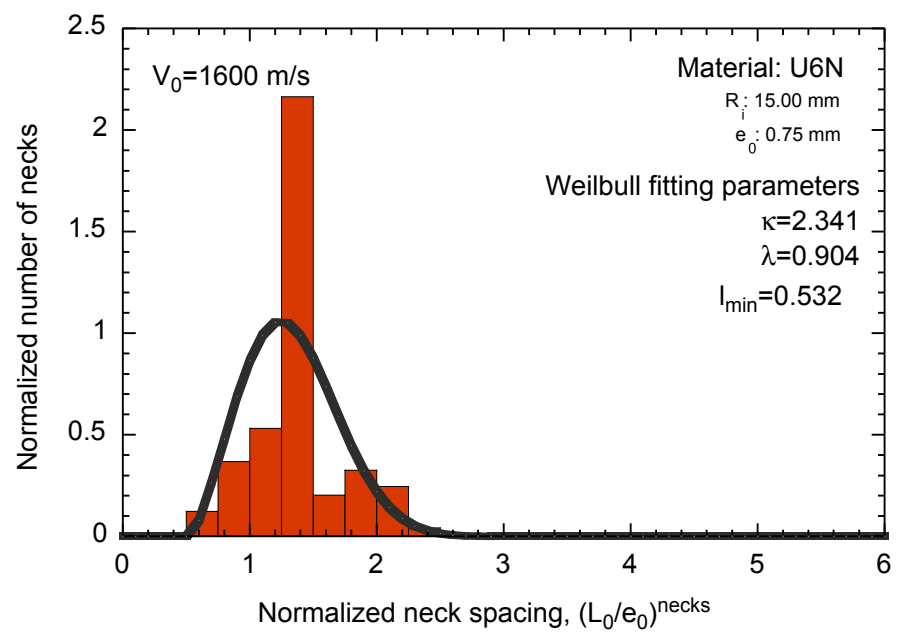

Fig. 7. Finite element result. Normalized number of necks as a function of the normalized neck spacing $\left(L_{0} / e_{0}\right)^{\text {necks }}$. $V_{0}=1600 \mathrm{~m} / \mathrm{s}$. Fitting parameters in Eq. (3). $\kappa=$ 2.341, $\lambda=0.904$ and $l_{\min }=0.532$. Material: U6N.

and 7) which is consistent with the experimental observations reported by Zhang and Ravi-Chandar (2006). The mean neck spacing is $\left(L_{0} / e_{0}\right)^{\text {necks }} \approx 1.5$.

Saturation in the number of fragments sheds light on the mechanisms which reside behind fragmentation at very high loading velocities. When the number of fragments saturates the fragment size is rather constant suggesting that the fragmentation mechanisms may not be explained by the statistical fragmentation theory of Mott (1947) and subsequent developments of Grady and co-workers (1982, 1984, 1985, 2003). 


\subsection{Non-hardening materials}

In order to analyse the effect of the material description on the fragmentation process, ring expansion simulations are performed considering non-hardening materials. Strain and temperature dependences of the material are precluded. Rate independent and rate dependent behaviours are analysed, Table 2, being the material response defined by

$$
\sigma_{y}=\sigma_{0}\left(\frac{\dot{\varepsilon}^{p}}{\dot{\varepsilon}_{r}}\right)^{m}
$$

where $\sigma_{0}$ is the reference yield stress, $\dot{\varepsilon}_{r}$ is the reference strain rate and $m$ is the rate sensitivity exponent. Changing the constitutive law has two advantages: (1) it permits to evaluate the suitability of the dimensionless ratio $L_{0} / e_{0}$ for analysis of the neck spacing, regardless the material description and (2) it facilitates further comparisons with the stability analysis as it will be shown in Section 5 . The dimensions of the ring are $R_{i}=15 \mathrm{~mm}$ in inner radius and $e_{0}=1.0 \mathrm{~mm}$ in thickness of square cross section. To be noted that the thickness of the ring has been increased with respect to previous configuration. This allows for analyzing the effect of the cross-section dimensions on the fragmentation. In other words, changing the thickness of the ring permits to evaluate the suitability of the dimensionless ratio $L_{0} / e_{0}$ for analysis of the neck spacing, regardless the ring dimensions. From this point on, the analysis relies on the reference material defined by the parameters of Table 2 unless otherwise noted.

Let us start the analysis considering the rate independent material, Table 2. In Fig. 8 is depicted the normalized mean fragment size and the normalized neck spacing upon loading velocity and initial strain rate. Again, once certain value of loading velocity is exceeded $\left(V_{0} \approx 1200 \mathrm{~m} / \mathrm{s}\right.$ in the present case) the number of fragments and the number of necks practically coincide. Interestingly, here is highlighted that the saturation value, $L_{0} / e_{0} \approx 1.5$, is that reported in Fig. 3; although in such a case the cross section of the ring showed different dimensions and the material definition was strain, rate and temperature dependent. To be pointed out that the development of a regular spacing between fragments, insensitive to variations in the material properties at sufficiently high loading velocities, was glimpsed by Sørensen and Freund (2000).

Next, the rate dependent material is considered in the computations, Table 2. Number of fragments and number of necks match at $V_{0} \approx 900 \mathrm{~m} / \mathrm{s}$. In comparison with the rate independent material, the loading velocity for which all the necks nucleated lead to failure locations is slightly smaller, however, the saturation value keeps close to $L_{0} / e_{0} \approx 1.5$.

Table 2

Parameter values characterizing rate independent and rate dependent material behaviours, Eq. (4).

\begin{tabular}{|c|c|c|c|}
\hline Symbol & Property and units & Rate independent material & Rate dependent material \\
\hline$\sigma_{0}$ & Reference yield stress (MPa), Eq. (4) & 500 & 500 \\
\hline$\dot{\varepsilon}_{r}$ & Reference strain rate $\left(\mathrm{s}^{-1}\right)$, Eq. (4) & - & 1000 \\
\hline$m$ & Rate sensitivity exponent, Eq. (4) & - & 0.01 \\
\hline $\begin{array}{l}\rho \\
E\end{array}$ & $\begin{array}{l}\text { Density }\left(\mathrm{kg} / \mathrm{m}^{3}\right) \\
\text { Young's modulus (GPa) }\end{array}$ & Initial strain rate, $\dot{\varepsilon}_{0} \underset{200}{7800}$ & $\begin{array}{l}7800 \\
200\end{array}$ \\
\hline
\end{tabular}

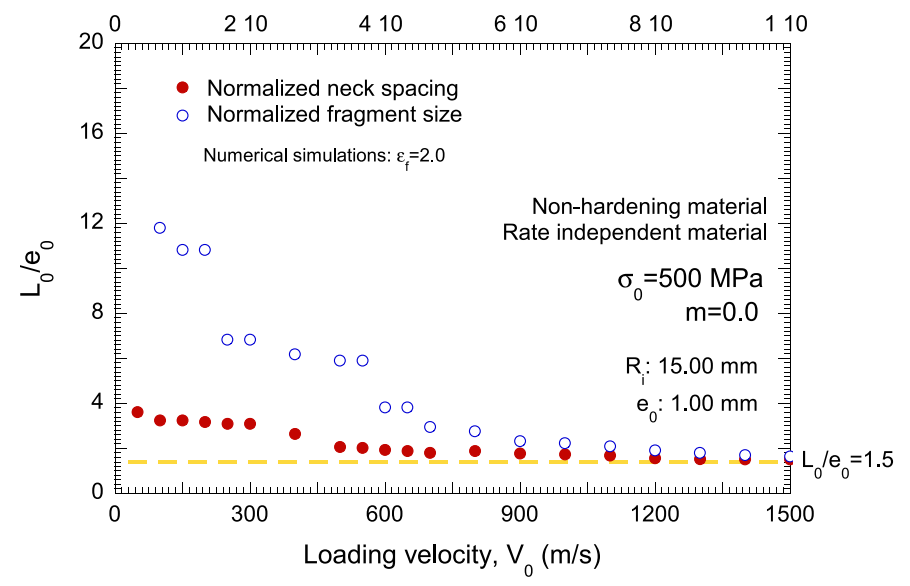

Fig. 8. Normalized mean fragment size and normalized neck spacing upon loading velocity $V_{0}$ and initial strain rate $\dot{\varepsilon}_{0}$. Rate independent material behaviour. Failure strain level $\varepsilon_{f}=2.0$. 
Next, a random perturbation is assigned to each node of the initial mesh of the ring modifying the position of the nodes in the unperturbed mesh to obtain a perturbed mesh framework. The purpose is to examine the role played by geometrical imperfections of small amplitude (additional to the numerical perturbations intrinsic to the FE analysis) on the saturation of the number of fragments. This is conducted by adding to the initial Lagrangian coordinates $\left(X_{0}, Y_{0}\right.$ and $\left.Z_{0}\right)$ of each node (unperturbed configuration) random initial displacements $(U, V, W)$ in each of the three directions.

The Lagrangian coordinates of the perturbed nodes in the whole mesh $(X, Y$ and $Z$ ) are then obtained following a Gaussian distribution characterized by a mean $s_{m}=0$ and a standard deviation $s_{d e v}$ of the form:

$$
\begin{aligned}
& X=X_{0}+U, \quad Y=Y_{0}+V, \quad Z=Z_{0}+W \\
& U, V, W: \text { random variables from a Gaussian distribution } N\left(0, s_{d e v}\right)
\end{aligned}
$$

Three different cases are analysed: $s_{d e v}=2 \times 10^{-6}, s_{d e v}=1 \times 10^{-5}$ and $s_{d e v}=2 \times 10^{-5}$ which correspond, respectively, to spatial variations of $0.1 \%, 0.5 \%$ and $1 \%$ of the distance between two adjacent nodes in the former position. The larger the standard deviation the greater the geometrical perturbation is.

In Fig. 10 is depicted the normalized mean fragment size upon loading velocity and initial strain rate for the former configuration (unperturbed configuration) and the three configurations for which the mesh has been perturbed. At low loading velocities the necking pattern is strongly dictated by the scatter of the geometrical perturbation, being the number of fragments sharply reduced with increasing standard deviation. Increasing loading velocity diffuses deformation throughout the material due to the emerging role played by inertia which delays flow localization. Then, the initial geometrical perturbation becomes less relevant in the process of necking nucleation. However, one should note that the larger the standard deviation the larger the mean fragment size even for the highest loading velocity considered. Therefore, inertia is not able to suppress completely the role played by the mesh perturbation on the fragments size. This behaviour can be explained keeping in mind that the initial imperfections have been introduced by perturbing the displacement field at the nodes. Thus, the wavelength associated to these imperfections is of the order of the mesh-size rather short wavelengths - and therefore inertia effects may not have enough influence on them.

Up to here, the analysis suggests the existence of a critical wavelength which may be responsible for the fragmentation at very high strain rates when inertia effects become dominant. The normalized critical wavelength $\left(L_{0} / e_{0}\right)^{\text {critical }}$ may show little dependence on the material description, on the dimensions of the sample cross section as well as on geometrical perturbations of small amplitude.

\section{Unitary periodic cell}

\subsection{Formulation}

Following the computational model developed by Xue et al. (2008) for assessing the neck development of an infinite plate under plane strain constraint, a circular cross section ring subjected to a radial expansion and with geometrical periodic perturbations can be modelled as a periodic collect of unitary axisymmetric cells with sinusoidal spatial imperfections following the expression:

$$
\phi=\phi_{0}\left(1-\frac{\delta}{2} \cos \left(\frac{2 \pi X_{1}}{L_{0}}\right)\right)
$$

being $\delta$ the amplitude of the imperfection, $L_{0}$ the initial length of the cell, $X_{1}$ the axial location of a point in the undeformed configuration, and $\phi_{0}=1 \mathrm{~mm}$ and $\phi$ the initial unperturbed and perturbed cross diameter of the cell, respectively. Due to the symmetry of the problem, only half of the cell needs to be analysed, Fig. 11.

Initial and boundary conditions are imposed corresponding to an initial axial loading rate $\dot{\varepsilon}_{0}=V_{0} / L_{0}$ :

$$
\begin{aligned}
& v_{1}\left(X_{1}, X_{2}, 0\right)=\dot{\varepsilon}_{0} X_{1} \\
& v_{2}\left(X_{1}, X_{2}, 0\right)=-\frac{\dot{\varepsilon}_{0}}{2} X_{2} \\
& u_{1}\left(0, X_{2}, t\right)=0 \\
& v_{1}\left(L_{0} / 2, X_{2}, t\right)=V_{0} / 2
\end{aligned}
$$

Being $v_{1}$ and $v_{2}$ the components of velocity in $X_{1}$ and $X_{2}$ directions, respectively, and $u_{1}$ the component of displacement in $X_{1}$ direction.

In order to avoid the abrupt jump in the stress field caused by application of the initial and boundary conditions previously described, the material flow has been initialized in the whole domain with a value of stress in direction $X_{1}$ equal to $\sigma=\sigma_{0}\left(\dot{\varepsilon}_{0} / \dot{\varepsilon}_{r}\right)^{m}$, Eq. (4). If neither the velocity nor the stress fields were initialized, for sufficiently high velocities the generated wave could induce by itself a neck (Needleman, 1991; Xue et al., 2008).

The axisymmetric unitary cell is numerically solved using ABAQUS/Explicit (2010). Four-node axisymmetric elements with reduced integration are used (CAX4R in ABAQUS notation). For all cases, near-square elements are uniformly distributed through the cell corresponding with a value of fifty elements along the radius of the revolution model. As aforementioned for the 3D numerical model of the ring expansion configuration, hourglass deformation modes have been 
prevented using the integral viscoelastic approach available in ABAQUS/Explicit (2010). The rate dependent material configuration is considered in the computations, Table 2.

\subsection{Results}

The amplitude of the imperfection is $\delta=0.0513$, Fig. 11. According to Xue et al. (2008) localized necking is assumed to start when $d \varepsilon_{B} / d t=0$, where $\varepsilon_{B}$ is the strain measured at the side $B$ of the sample as illustrated in Fig. 11 and $t$ refers to the loading time. In other words, localized necking describes the stage of the loading process for which plastic flow is fully concentrated in the neck region.

In Fig. 12 the localized necking strain is computed over a range of imperfection cell lengths for different loading rates. Small ratios $L_{0} / \phi_{0}$ lead to large necking strains, which are practically independent of the loading rate. This is consistent with the considerations reported by Mercier and Molinari (2003) who demonstrated that stress multiaxial effects damp the short wavelengths. Moreover, large ratios $L_{0} / \phi_{0}$ lead to large necking strains at high strain rates. This agrees with the conclusions derived by Fressengeas and Molinari (1994) who demonstrated that inertia is responsible for slowing down the growth of long wavelengths. Combination of the stabilizing aspects of multidimensional effects on short wavelengths and the stabilizing aspects of inertia on long wavelengths leads to the promotion of intermediate wavelengths which determine the minimum necking strain, Fig. 12. Then, this minimum necking strain becomes larger with increasing loading rate due to the emerging role of inertia. The concept of the cell length associated to the minimum necking strain is tied to the minimum investment of energy required for the necking formation. Here should be noted that the cell length associated to the minimum necking strain decreases with the loading rate running asymptotically to $L_{0} / \phi_{0} \approx 1.5$. This value is in close agreement with the aspect ratio $L_{0} / e_{0}$ which dictated the saturation of the number of necks and fragments in the 3D simulations of the ring expansion problem reported in previous section, Figs. 3, 8 and 9.

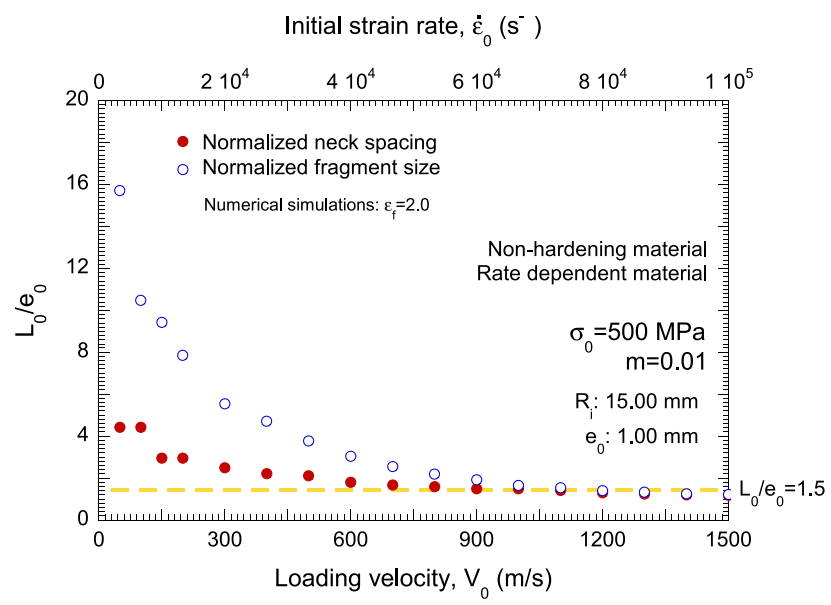

Fig. 9. Normalized mean fragment size and normalized neck spacing upon loading velocity $V_{0}$ and initial strain rate $\dot{\varepsilon}_{0}$. Rate dependent material behaviour, Table 2. Failure strain level $\varepsilon_{f}=2.0$.

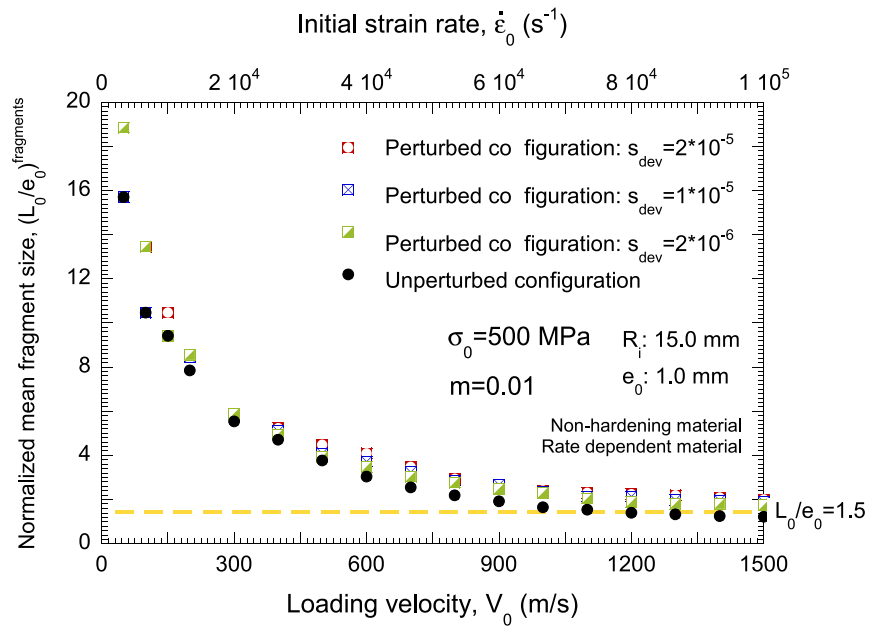

Fig. 10. Normalized mean fragment size upon loading velocity $V_{0}$ and initial strain rate $\dot{\varepsilon}_{0}$ for the former configuration and the three configurations for which the mesh has been perturbed. Rate dependent material behaviour. Failure strain level $\varepsilon_{f}=2.0$. 


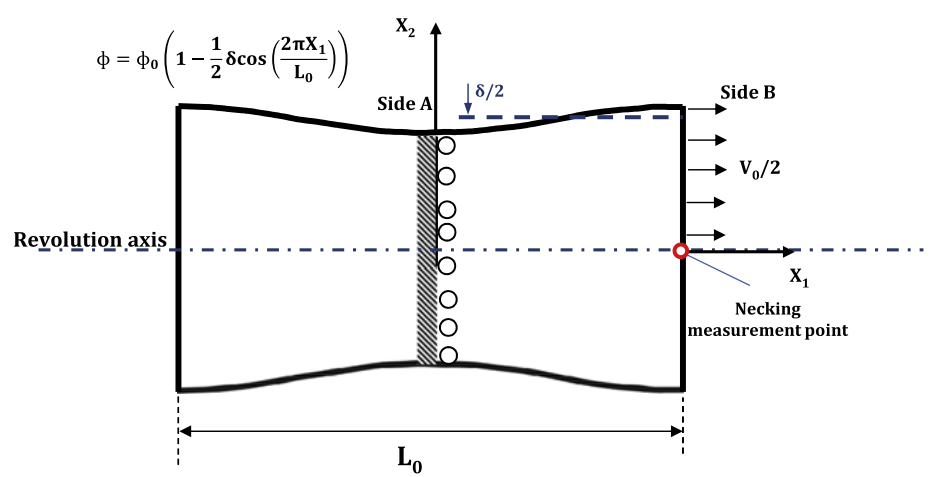

Fig. 11. Cell model of an axisymmetric tensile specimen with a small geometric defect near $X_{1}=0$.

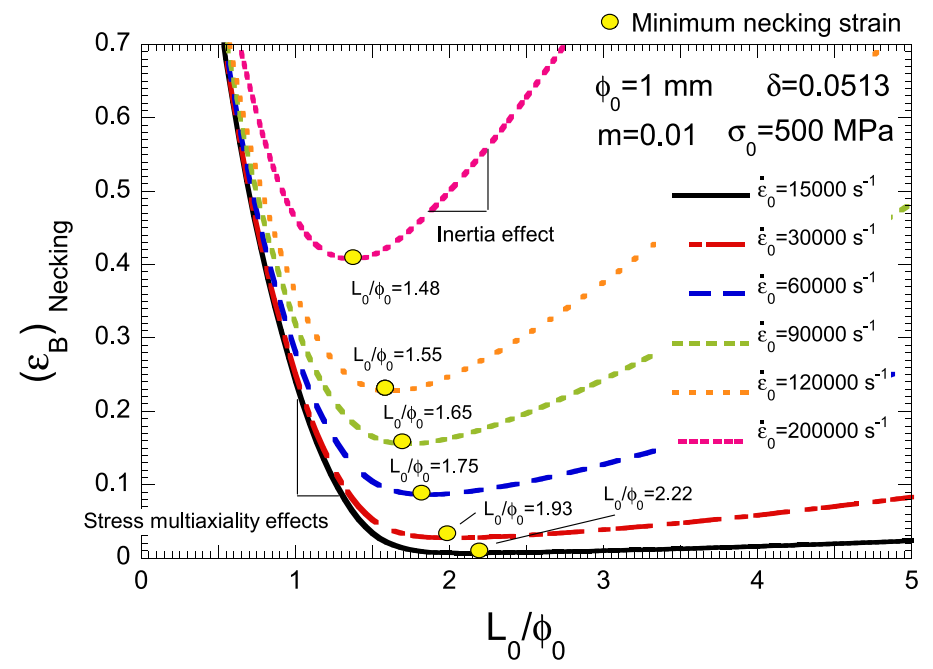

Fig. 12. Localized necking strain $\left(\varepsilon_{B}\right)_{\text {Necking }}$ upon the cell length $L_{0} / \phi_{0}$ for different initial loading rates $\varepsilon_{0}^{\cdot}$ where $\varepsilon_{0}^{\cdot}=V_{0} / L_{0}$. Imperfection amplitude: $\delta=0.0513$. Imperfection amplitude: $\delta=0.0513$. Rate dependent material, Table 2 .

Inertia not only retards the onset of localized necking but also plays the role slowing down strain localization (Knoche and Needleman, 1993; Han and Tvergaard, 1995). This is illustrated in Fig. 13 where the evolution of the force measured at side B of the cell, see Fig. 11, as a function of the loading time for $\dot{\varepsilon}_{0}=90000 \mathrm{~s}^{-1}$ and two different wavelengths, $L_{0} / \phi_{0}=2.6$ and $L_{0} / \phi_{0}=4.0$, is depicted. The numerical results are compared with the homogeneous fundamental solution given by

$$
F(t)=F(0)\left(\frac{L_{0}}{L_{0}+V_{0} t}\right)^{m+1}
$$

This fundamental solution assumes that the cross section and the deformation are uniform at any time (absence of any defect). Numerical computations and analytical solution find good agreement from the onset of loading up to the loading time corresponding to localized necking $d \varepsilon_{B} / d t=0$. This period, during which the cell undergoes a quasi-uniform elongation, is characterized by a monotonic decay of the mean force level upon time since the material does not strainharden. Once the condition $d \varepsilon_{B} / d t=0$ is fulfilled the numerical solution deviates significantly from the fundamental homogeneous solution. This deviation is a manifestation of localized necking. From this point on, the role played by inertia on slowing down the development of the instability becomes evident.

In the case of $L_{0} / \phi_{0}=2.6$ the post-uniform elongation regime is characterized by a pronounced drop of the force. The plastic flow rapidly localizes in a narrow zone at the middle of the cell, triggering material failure in a short period of time. Comparatively, the instability development is considerably slowed down in the case $L_{0} / \phi_{0}=4.0$.

The stabilizing effects of inertia in the post-uniform elongation regime can be understood by plotting the number of localization points formed upon the cell wavelength, Fig. 14. Within the range of wavelengths less than or equal to the critical one $\left(L_{0} / \phi_{0}\right)^{\text {critical }} \approx 1.5$, only a localization point is developed in the cell, no matter the loading rate value. However, once the critical cell length is exceeded, it is feasible that the number of localization points formed in the cell in the postuniform elongation regime will be larger than one. This occurs if the strain rate is high enough for inertia to play a key role in the loading process. Then, inertia induces a sharp minimum in the $\varepsilon_{B}-L_{0} / \phi_{0}$ diagram, Fig. 12 . In such a case the number 

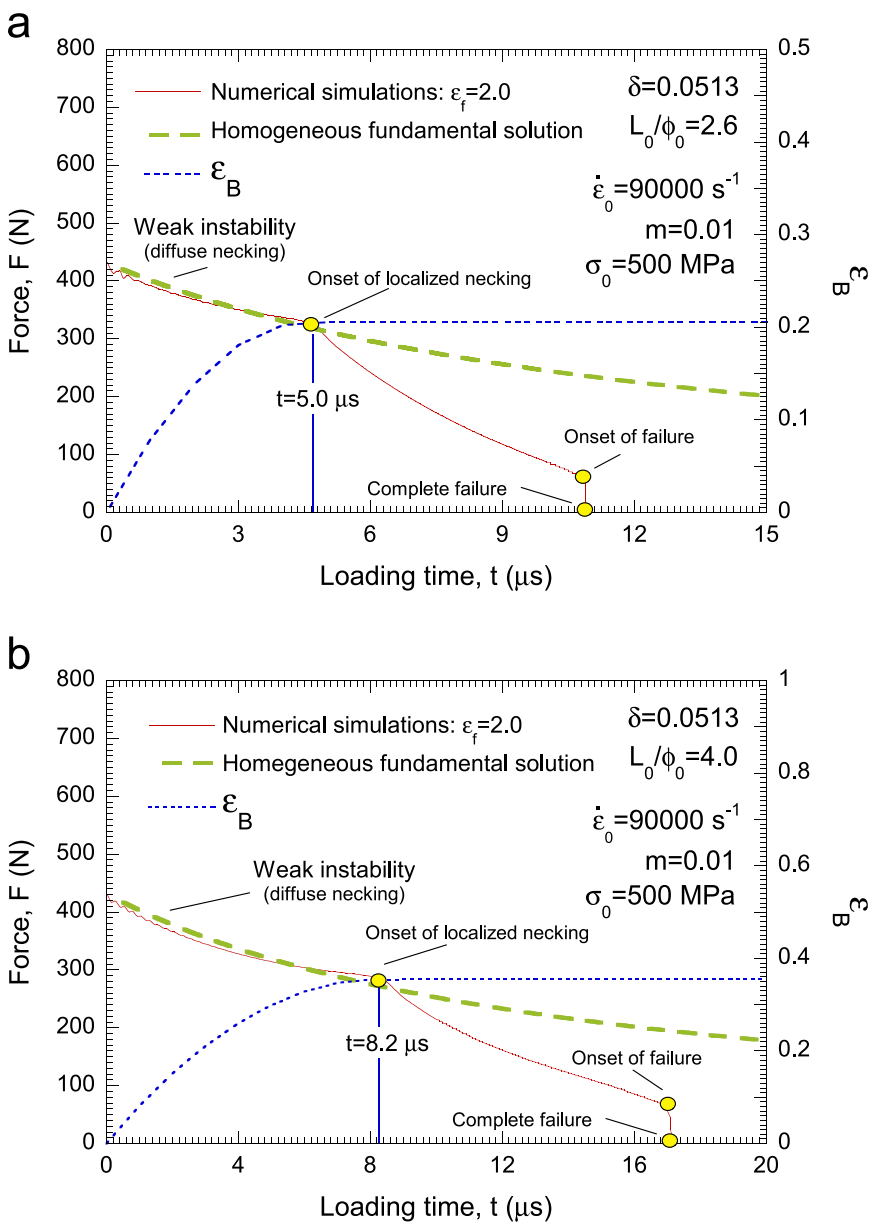

Fig. 13. Force $F(t)$ and strain $\varepsilon_{B}(t)$ upon loading time $t$ for $\varepsilon_{0}^{\cdot}=90000 \mathrm{~s}^{-1}$. Imperfection amplitude: $\delta=0.0513$. (a) $L_{0} / \phi_{0}=2.6$, (b) $L_{0} / \phi_{0}=4.0$. Rate dependent material. Failure strain $\varepsilon_{f}=2.0$.

of localization points formed tends to correlate with the ratio $\left(L_{0} / \phi_{0}\right) /\left(L_{0} / \phi_{0}\right)^{\text {critical }}$ where $L_{0} / \phi_{0}$ is the aspect ratio of the considered cell and $\left(L_{0} / \phi_{0}\right)^{\text {critical }}$ is the aspect ratio of the cell corresponding to the minimum necking strain. This reinforces the idea of the existence of a critical wavelength which dictates the development of multiple necking in very high loading processes. Following this idea, it should be noted here that some computations showed the formation of an even number of localization points. In other words, under certain loading conditions the material location corresponding to the maximum imperfection amplitude does not evolve as a localization point, the geometrical imperfection is vanished by the stabilizing effect of inertia and the mean distance between localization points follows the critical cell length, see Fig. 14 .

Thus, the analysis is extended to different imperfection amplitudes within the range $0.01 \leq \delta \leq 0.1$. Fig. 15 shows the necking strain $\left(\varepsilon_{B}\right)_{\text {Necking }}$ as a function of the cell length $L_{0} / \phi_{0}$ for different imperfection amplitudes $\delta$ at high strain rate $\dot{\varepsilon}_{0}=120000 \mathrm{~s}^{-1}$. A drastic rise in the minimum necking strain is observed by decreasing one order of magnitude the imperfection amplitude. However, such variation in the size of the defect involves only a small change in the critical cell length associated to the minimum necking strain. The amplitude of the imperfection significantly affects the stress multiaxiality effects enhancing the role played by the hydrostatic pressure in the necked region, however, the contribution of inertia seems to remain rather unaffected. Thus, the critical wavelength that minimizes the necking strain increases with imperfection amplitude.

\section{Linear perturbation analysis}

\subsection{Formulation}

Alternatively, the equations governing the stretching process of the expanding ring are derived within a quasi-1D theoretical framework.

Details of the formulation can be found in Zhou et al. (2006c) and Vadillo et al. (2012), but we present here the main equations for completeness. The ring expansion process is approximated to a cylindrical bar with cross-section radius $r_{0}$, area $A_{0}=\pi r_{0}^{2}$, and length $L$ subjected to axial velocity $V_{0}$ and uniform tensile strain rate $\dot{\varepsilon}$. At time $t$ and for any cross 
Rate dependent material $-\sigma_{0}=500 \mathrm{MPa}, \mathrm{m}=0.01, \delta=0.0513$

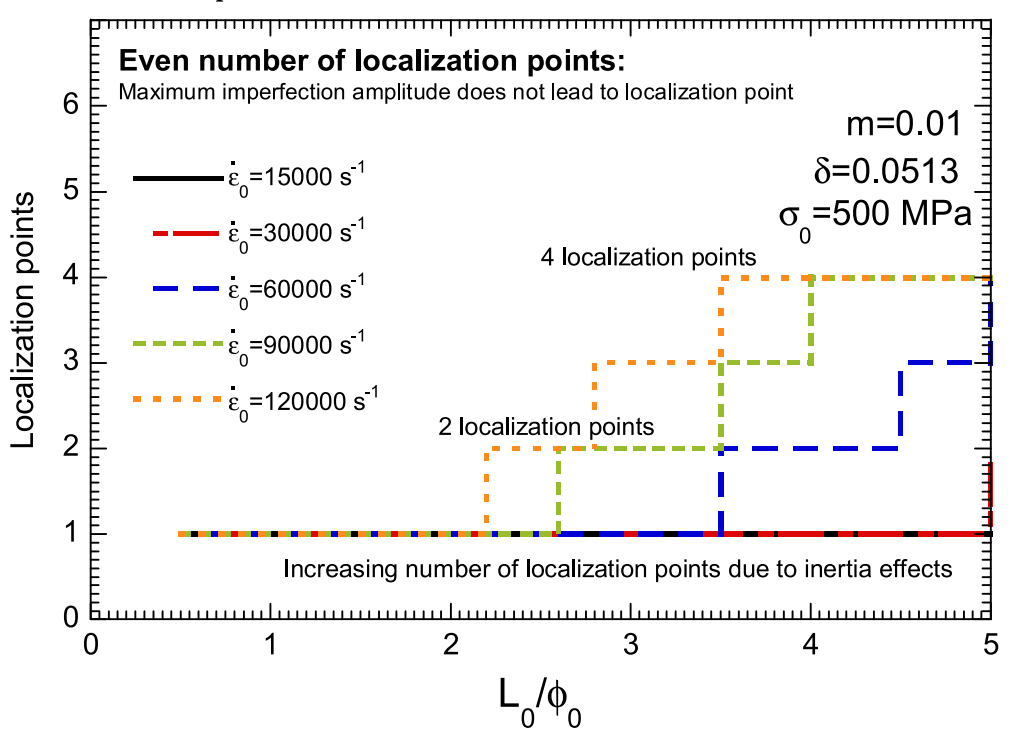

Strain rate contours - Initial loading rate, $\dot{\varepsilon}_{0}=90000 \mathrm{~s}^{-1}$

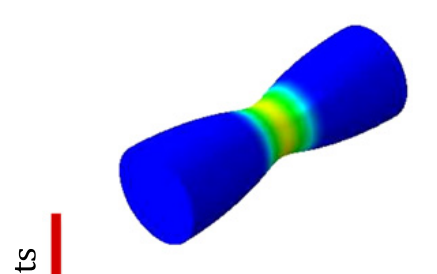

$\mathrm{L}_{0} / \phi_{0}=1.75$

Loading time, $\mathrm{t}=610^{-6} \mathrm{~s}$

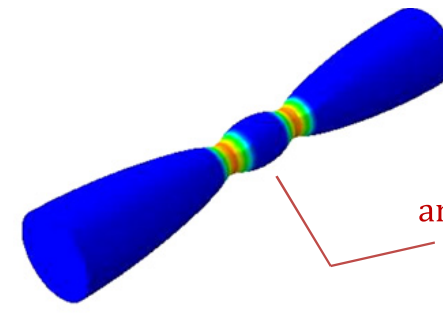

$\mathrm{L}_{0} / \phi_{0}=2.6$

Loading time, $\mathrm{t}=1.110^{-5} \mathrm{~s}$

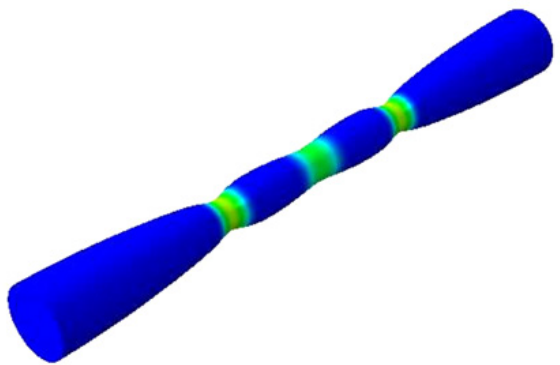

$$
\mathrm{L}_{0} / \phi_{0}=3.5
$$

Loading time, $\mathrm{t}=1.410^{-5} \mathrm{~s}$

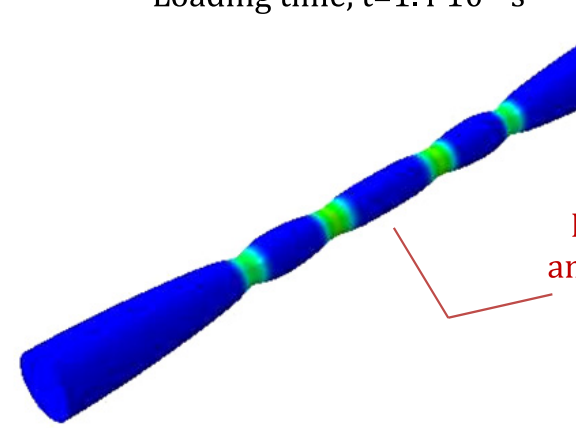

$$
\mathrm{L}_{0} / \phi_{0}=4
$$

Loading time, $\mathrm{t}=1.710^{-5} \mathrm{~s}$
Maximum imperfection amplitude does not lead to localization point

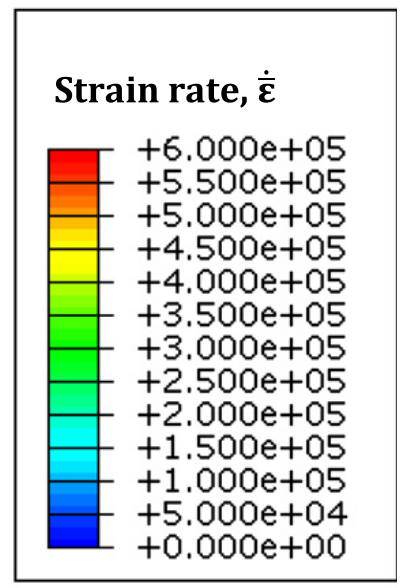

Maximum imperfection amplitude does not lead to localization point

Fig. 14. Number of localization points as a function of the cell length $L_{0} / \phi_{0}$. Imperfection amplitude: $\delta=0.0513$. Rate dependent material. 


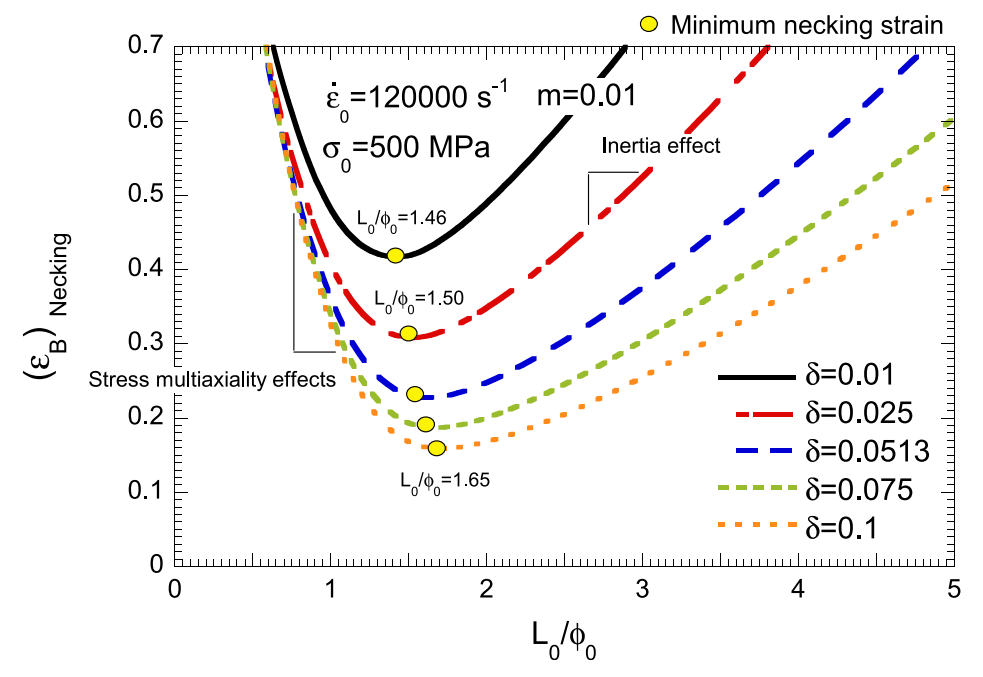

Fig. 15. Necking strain $\left(\varepsilon_{B}\right)_{\text {Necking }}$ as a function of the cell length $L_{0} / \phi_{0}$ for different imperfection amplitudes $\delta$. Initial strain rate $\dot{\varepsilon}_{0}=120000 \mathrm{~s}^{-1}$. Rate dependent material.

section of the bar, $X$ is the Lagrangian coordinate $X(0 \leq X \leq L), x$ is the Eulerian coordinate, $v$ the current axial velocity, $r$ and $A$ the current radius and cross-sectional area, respectively, and $\sigma$ the true stress. The fundamental equations of the loading process are presented as follows:

$$
\left\{\begin{array}{l}
\left(\frac{\partial v}{\partial X}\right)_{t}=e^{\varepsilon} \dot{\varepsilon} \\
\rho A_{0}\left(\frac{\partial v}{\partial t}\right)_{X}=A\left(\frac{\partial \sigma}{\partial X}\right)_{t}+\sigma\left(\frac{\partial A}{\partial X}\right)_{t} \\
A=A_{0} e^{\varepsilon} \\
\sigma_{y}=\Psi(\varepsilon, \dot{\varepsilon}) \\
\sigma=\left(1+\theta^{-1}\right) \ln (1+\theta) \sigma_{y} \\
\theta=\frac{1}{2} r\left(\frac{\partial^{2} r}{\partial x^{2}}\right)=\frac{2 A\left(\partial^{2} A / \partial x^{2}\right)-(\partial A / \partial x)^{2}}{8 \pi A}
\end{array}\right.
$$

where $\varepsilon=\ln \left[(\partial x / \partial X)_{t}\right], \dot{\varepsilon}=(\partial \varepsilon / \partial t), \sigma_{y}=\Psi(\varepsilon, \dot{\varepsilon})$ is the material yield stress and $\theta$ is a geometrical parameter which gathers the stress multiaxial effects tied to necked section (Bridgman, 1952; Walsh, 1984; Fressengeas and Molinari, 1985).

Now let us set the initial and boundary conditions for a bar subjected to constant and uniform strain rate:

$$
v(X, 0)=\dot{\varepsilon}_{0} X, \quad V_{0}(0, t)=0, \quad v(L, t)=\dot{\varepsilon}_{0} L
$$

According to Eq. (10), the system of equations given by Eq. (9) has the following homogeneous solution:

$$
f_{1}=\left(v_{1}(X), \varepsilon_{1}(t), \dot{\varepsilon}_{1}(t), A_{1}(t), r_{1}(t), \sigma_{1}(t), \theta_{1}\right)^{T}
$$

This defines the background state of the boundary value problem in the absence of flow instability.

A linear perturbation solution is intended adding the following small perturbation to the fundamental solution defined by Eq. (11) at a given time $t_{0}$ (Zhou et al., 2006c):

$$
\delta f e^{i \xi X}=(\delta v, \delta \varepsilon, \delta \dot{\varepsilon}, \delta A, \delta r, \delta \theta, \delta \sigma)^{T} e^{i \xi X}
$$

In previous expression $\xi$ is the wavenumber and $(\delta v, \delta \varepsilon, \delta \dot{\varepsilon}, \delta A, \delta r, \delta \theta, \delta \sigma)$ are the differences between the actual perturbed solution and the homogeneous solution.

Then, at a time $t>t_{0}$ the perturbed solution of the aforementioned system of equations has the following form:

$$
f=f_{1}+\delta f e^{i \xi X+\eta\left(t-t_{0}\right)}
$$

where $\eta$ is the perturbation growth rate which is assumed constant with respect to time. According to Fressengeas and Molinari (1985) this is consistent for perturbations whose variation is much faster than that of the background solution.

Introducing Eq. (13) into Eq. (9) and keeping only the first-order terms, a linear homogeneous system to obtain $\delta f$ is derived.

A non-trivial solution for $\delta f$ can be derived if the determinant of the aforementioned system of algebraic linear equations is equal to zero. This leads to the following quadratic expression in $\eta$ :

$$
\eta^{2}+\left[\dot{\varepsilon}_{1}\left(t_{0}\right)+\frac{\xi^{2}}{\rho_{0}} e^{-2 \varepsilon_{1}\left(t_{0}\right)} \frac{\partial \Psi}{\partial \dot{\varepsilon}}\right] \eta+\frac{\xi^{2}}{\rho_{0}} e^{-2 \varepsilon_{1}\left(t_{0}\right)}\left[\frac{\partial \Psi}{\partial \varepsilon}+\sigma_{1}\left(t_{0}\right)\left(\frac{A_{0} e^{-3 \varepsilon_{1}\left(t_{0}\right)}}{8 \pi} \xi^{2}-1\right)\right]=0
$$


At this point, the following dimensionless variables are introduced (Zhou et al., 2006c; Vadillo et al., 2012):

$$
\begin{aligned}
& \bar{\eta}=\frac{\eta}{\dot{\varepsilon}_{1}}, \quad \bar{\xi}=r_{0} \xi \\
& \Psi(\varepsilon, \dot{\varepsilon})=\sigma_{0} \psi(\varepsilon, \dot{\varepsilon}), \quad \bar{L}=\frac{\sqrt{\sigma_{0} / \rho}}{r_{0}} \frac{\sqrt{\psi\left(\varepsilon_{1}, \dot{\varepsilon}_{1}\right)}}{\dot{\varepsilon}_{1}} \\
& \chi_{m}=\left(\frac{\dot{\varepsilon}_{1}}{\psi\left(\varepsilon_{1}, \dot{\varepsilon}_{1}\right)}\right) \cdot \frac{\partial \psi(\varepsilon, \dot{\varepsilon})}{\partial \dot{\varepsilon}}, \quad \chi_{n}=\left(\frac{1}{\psi\left(\varepsilon_{1}, \dot{\varepsilon}_{1}\right)}\right) \cdot \frac{\partial \psi(\varepsilon, \dot{\varepsilon})}{\partial \varepsilon}
\end{aligned}
$$

where $\bar{\eta}$ is the dimensionless perturbation growth, $\bar{\xi}$ is the dimensionless wavenumber, $\sigma_{0}$ is a reference yield stress, $\psi(\varepsilon, \dot{\varepsilon})$ is the dimensionless material yield stress, $\bar{L}$ is a dimensionless material parameter which gathers geometrical and material effects, $\chi_{m}$ is the logarithmic rate sensitivity and $\chi_{n}$ is the dimensionless strain hardening parameter. It has to be noted that in a dimensionless form of the equation of movement for the ring expansion problem, $\bar{L}^{2}$ is the factor multiplying the acceleration, thus representing the inertial resistance to motion. As $\bar{L}$ decreases the material behavior is stabilized, delaying the formation and development of plastic instabilities.

Then, with the new dimensionless variables, Eq. (14) takes the following form:

$$
\left.\bar{\eta}^{2}+\left(1+\Lambda^{2} \chi_{m} \bar{L}^{2} \bar{\xi}^{2}\right) \bar{\eta}+\bar{L}^{2} \bar{\xi}^{2} \Lambda^{2} \quad \chi_{n}+\Lambda^{3} \frac{\bar{\xi}^{2}}{8}-1\right)=0
$$

where $\Lambda=e^{-\varepsilon_{1}\left(t_{0}\right)}$.

In generalized form, the condition for the perturbation growth is $\bar{\xi}<(2 / \Lambda) \sqrt{2\left(1-\chi_{n}\right) / \Lambda}$. If this condition is fulfilled the perturbation growth is defined by

$$
\bar{\eta}^{+}=\frac{-\left(1+\Lambda^{2} \chi_{m} \bar{L}^{2} \bar{\xi}^{2}\right)+\sqrt{\left(1+\Lambda^{2} \chi_{m} \bar{L}^{2} \bar{\xi}^{2}\right)^{2}+4 \bar{L}^{2} \Lambda^{2} \bar{\xi}^{2}\left(1-\chi_{n}-\Lambda^{3} \frac{\bar{\xi}^{2}}{8}\right)}}{2}
$$

Imposing $\partial \bar{\eta}^{+} / \partial \bar{\xi}=0$ in Eq. (17) the dominant wavenumber $\bar{\xi}_{c}$ and the critical perturbation growth $\bar{\eta}_{c}^{+}$are determined as

$$
\begin{gathered}
\bar{\xi}_{c}=\left[\frac{2 \Lambda\left(2+\chi_{m}-2 \chi_{n}\right)-2 \chi_{m} \sqrt{\Lambda\left(\Lambda+8 \bar{L}^{2}\left(1-\chi_{n}\right)\left(1-\chi_{n}+\chi_{m}\right)\right)}}{\Lambda^{3}\left(1-2 \bar{L}^{2} \chi_{m}^{2}\right)}\right]^{0.5} \\
\bar{\eta}_{c}^{+}=\bar{\eta}^{+}\left(\bar{\xi}_{c}\right)
\end{gathered}
$$

To be consistent with the analysis derived in previous sections, non-hardening materials are considered $\chi_{n}=0$. Then, the condition for the perturbation growth becomes $\bar{\xi}<(2 / \Lambda) \sqrt{2 / \Lambda}$ and Eq. (17) can be simplified as

$$
\bar{\eta}^{+}=\frac{-\left(1+\Lambda^{2} \chi_{m} \bar{L}^{2} \bar{\xi}^{2}\right)+\sqrt{\left(1+\Lambda^{2} \chi_{m} \bar{L}^{2} \bar{\xi}^{2}\right)^{2}+4 \bar{L}^{2} \Lambda^{2} \bar{\xi}^{2}\left(1-\Lambda^{3} \frac{\bar{\xi}^{2}}{8}\right)}}{2}
$$

Leading to the following definition of the dominant wavenumber $\bar{\xi}_{c}$

$$
\bar{\xi}_{c}=\left[\frac{2 \Lambda\left(2+\chi_{m}\right)-2 \chi_{m} \sqrt{\Lambda\left(\Lambda+8 \bar{L}^{2}\left(1+\chi_{m}\right)\right)}}{\Lambda^{3}\left(1-2 \bar{L}^{2} \chi_{m}^{2}\right)}\right]^{0.5}
$$

\subsection{Results}

The above mentioned linear perturbation method is applied to the ring expansion analysis previously approached with the 3D ring expansion computations and the simulations of the unitary periodic cell. We consider that the instability process (onset on necking) starts at $t=0(\Lambda=1)$. Then, from Eq. (19) the perturbation growth as a function of the normalized perturbation wavelength $\left(L_{0} / \phi_{0}\right)$ for different values of initial strain rate, $\dot{\varepsilon}_{0}$, is plotted in Fig. 16 for the rate dependent material. Note that the normalized wavelength is related to the dimensionless wavenumber $\bar{\xi}$ through $L_{0} / \phi_{0}=\pi / \bar{\xi}$. From this figure it can be seen that, irrespective to material properties (Vadillo et al., 2012), the critical value of $L_{0} / \phi_{0}$ (corresponding to the maximum of $\bar{\eta}^{+}$) tends asymptotically to $\pi / 2$. Here should be noted that this value agrees with the results obtained from the 3D ring expansion simulations and the finite element modelling of the unitary periodic cell.

The selection of $t=0$ as the onset of necking time is consistent with the high level of $\bar{\eta}_{c}^{+}$observed initially for all the values of $\dot{\varepsilon}_{0}$ considered here (always greater than 5 for $m=0.01$ and even larger values for $m=0$ ). A value of the normalized growth rate $\bar{\eta}_{c}^{+}$larger than 5 can be considered as indicative of a well developed instability process. It should be noted, 


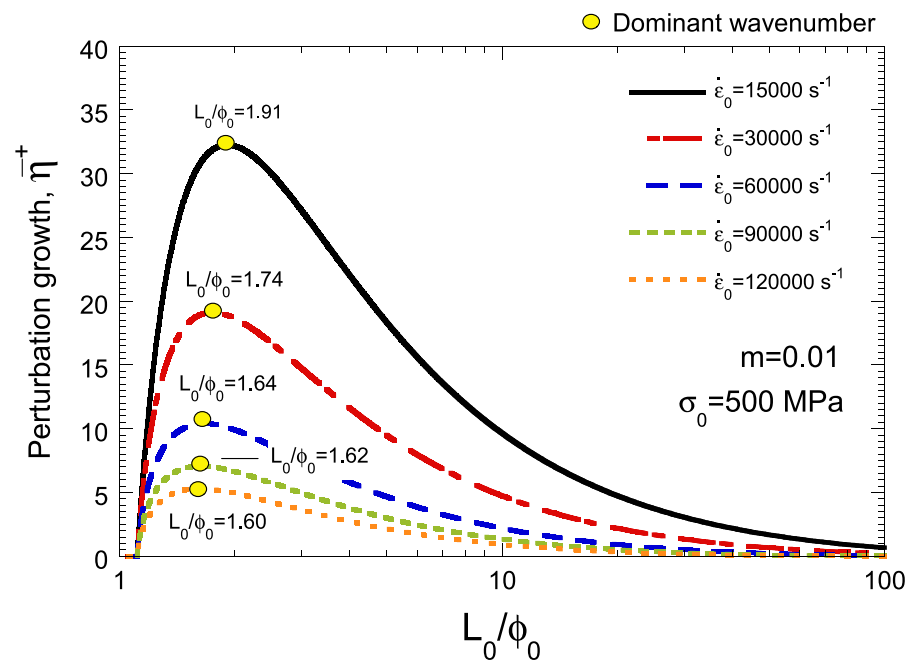

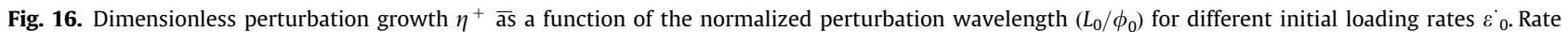
dependent material.

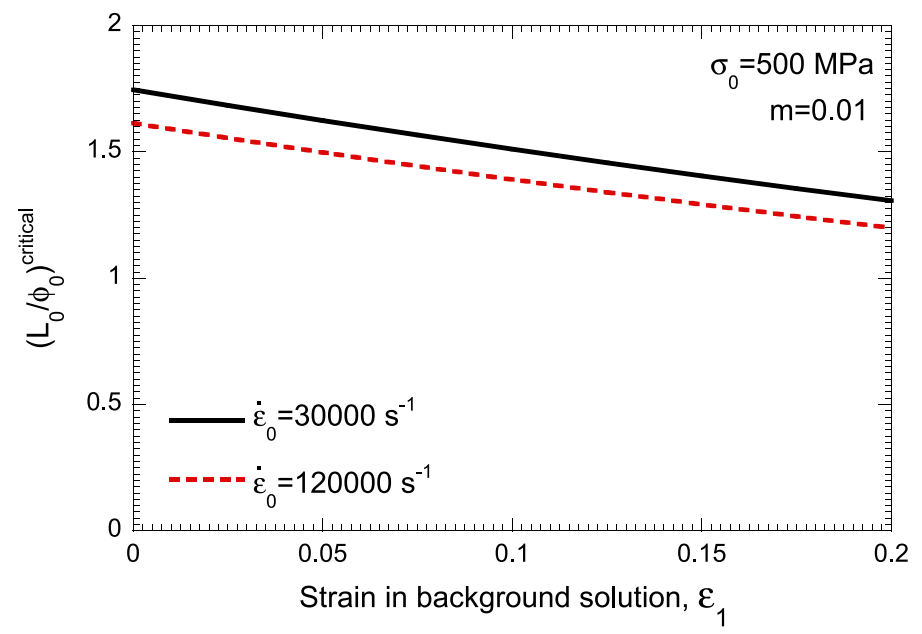

Fig. 17. Critical normalized neck spacing $\left(L_{0} / \phi_{0}\right)^{\text {critical }}$ as a function of the strain of the background solution $\varepsilon_{1}$ for initial strain rates $\varepsilon_{0}{ }_{0}=30000 \mathrm{~s}^{-1}$ and $\varepsilon_{0}{ }_{0}=120000 \mathrm{~s}^{-1}$. Rate dependent material.

that the rate of growth of perturbations is quite reduced for values of the rate sensitivity exponent a bit larger than $m=0.01$. In this case, the neck spacing determined at initial time by the linear stability analysis does not accurately represent the neck pattern observed in the sample at later times.

We have checked for $m=0.01$ the effect of the evolution of strain on the results. The value of the dimensionless critical wavelength $\left(L_{0} / \phi_{0}\right)^{\text {critical }}$ has been calculated in Fig. 17 in terms of the strain of the background solution $\varepsilon_{1}$.

For a given initial strain rate, the critical spacing between necks decreases with $\varepsilon_{1}$. This relation justifies values of $\left(L_{0} / \phi_{0}\right)^{\text {critical }}$ slightly smaller than $\pi / 2$ as those reported in Fig. 15.

\section{Discussion}

The purpose is to combine the results obtained so far by the three different approaches (3D simulations, unitary cell simulations and stability analysis) in order to provide an overview of the governing role played by the wavelength in the fragmentation of ductile rings expanding at very high strain rates. It should be noted that here $\phi_{0}$ is used to designate indifferently the width of a square cross-section in the 3D simulations or the diameter of a circular-cross section in the unitary cell simulations and stability analysis. The amplitude of the imperfection for the unitary periodic cell is $\delta=0.0513$. Non-hardening materials are considered and the strain of the background solution in the perturbation analysis is set to 0 . Results are obtained for small values of the rate sensitivity $(m \leq 0.01)$ for which the perturbation growth-rate is quite large.

Fig. 18 depicts, upon loading velocity $V_{0}$ and initial strain rate $\dot{\varepsilon}_{0}$, the wavelength $L_{0} / e_{0}$ which characterizes the normalized neck spacing and the normalized mean fragment size in the fully 3D FE simulations of the ring expansion, the cell-length $L_{0} / \phi_{0}$ which characterizes the minimum necking strain in the FE simulations of the unitary periodic cell and 


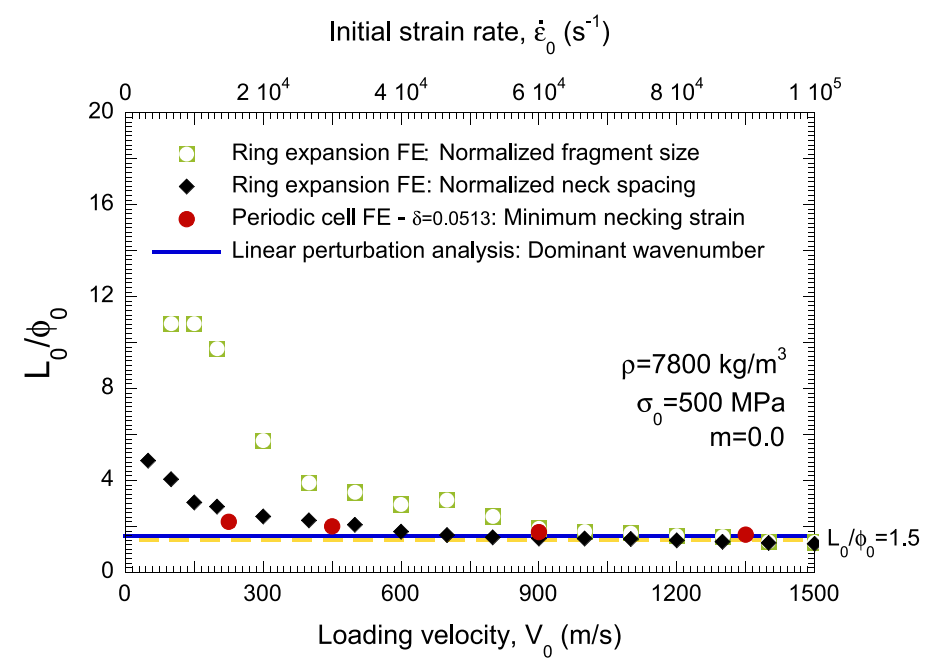

Fig. 18. The wavelength $L_{0} / e_{0}$ which characterizes the normalized neck spacing and the normalized mean fragment size in the fully 3D FE simulations of the ring expansion, the cell-length $L_{0} / \phi_{0}$ which characterizes the minimum necking strain in the FE simulations of the unitary periodic cell and the wavelength $L_{0} / \phi_{0}$ which characterizes the dominant wavelength in the linear stability analysis as a function of loading velocity $V_{0}$ and initial strain rate $\dot{\varepsilon}_{0}$. Rate independent material, Eq. (4) with $\rho=7800 \mathrm{~kg} / \mathrm{m}^{3}$ and $\sigma_{0}=500 \mathrm{MPa}$.

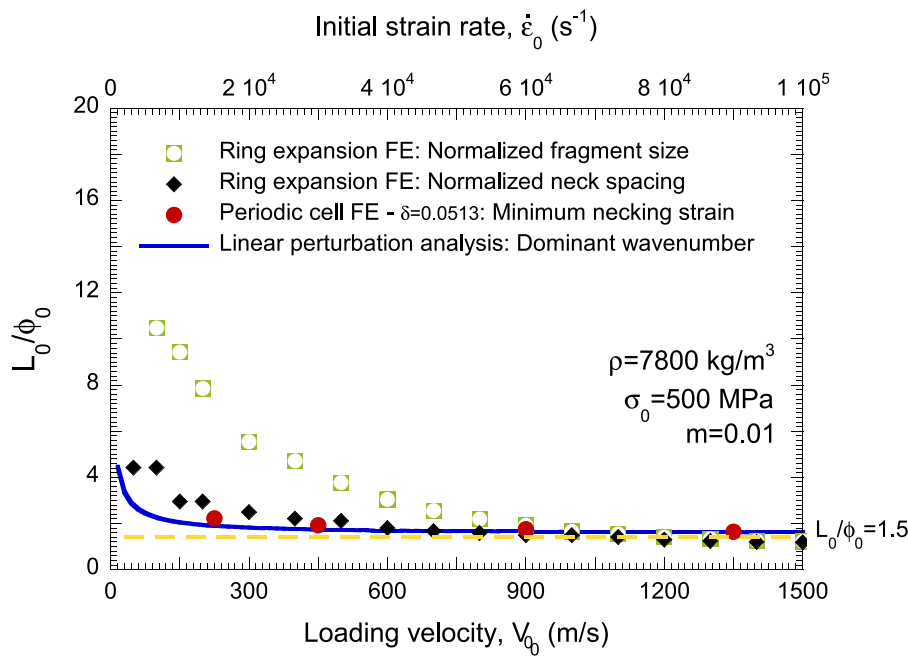

Fig. 19. The wavelength $L_{0} / e_{0}$ which characterizes the normalized neck spacing and the normalized mean fragment size in the fully $3 \mathrm{D}$ FE simulations of the ring expansion, the cell-length $L_{0} / \phi_{0}$ which characterizes the minimum necking strain in the FE simulations of the unitary periodic cell and the wavelength $L_{0} / \phi_{0}$ which characterizes the dominant wavelength in the linear stability analysis as a function of loading velocity $V_{0}$ and initial strain rate $\dot{\varepsilon}_{0}$. Rate dependent material, Eq. (4) with $\rho=7800 \mathrm{~kg} / \mathrm{m}^{3}, \sigma_{0}=500 \mathrm{MPa}, m=0.01$ and $\dot{\varepsilon}_{r}=1000 \mathrm{~s}^{-1}$.

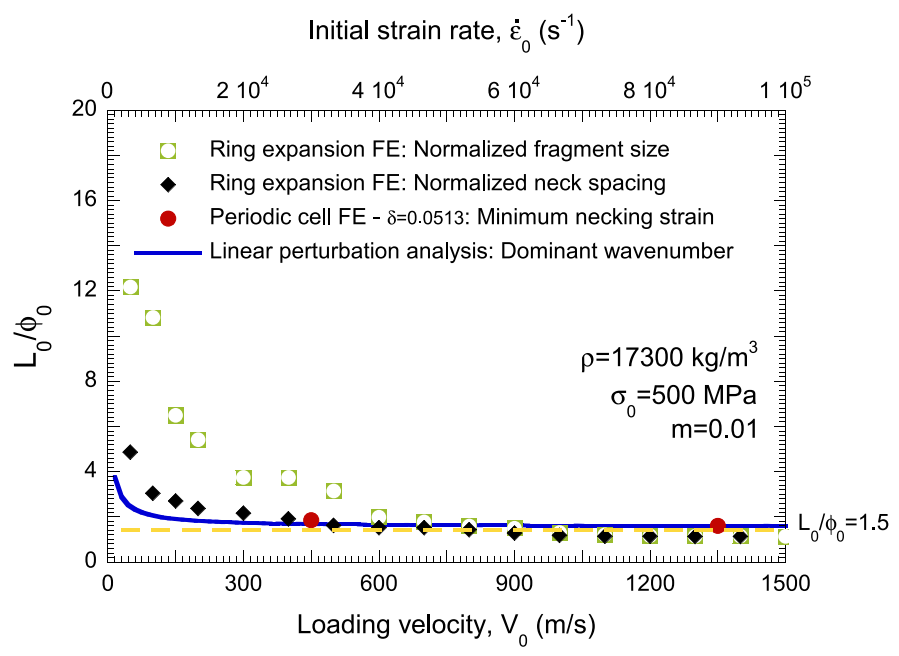

Fig. 20. The wavelength $L_{0} / e_{0}$ which characterizes the normalized neck spacing and the normalized mean fragment size in the fully 3D FE simulations of the ring expansion, the cell-length $L_{0} / \phi_{0}$ which characterizes the minimum necking strain in the FE simulations of the unitary periodic cell and the wavelength $L_{0} / \phi_{0}$ which characterizes the dominant wavelength in the linear stability analysis as a function of loading velocity $V_{0}$ and initial strain rate $\dot{\varepsilon}_{0}$. Rate dependent material, Eq. (4) with $\rho=17300 \mathrm{~kg} / \mathrm{m}^{3}, \sigma_{0}=500 \mathrm{MPa}, m=0.01$ and $\dot{\varepsilon}_{r}=1000 \mathrm{~s}^{-1}$. 
the wavelength $L_{0} / \phi_{0}$ which characterizes the dominant wavelength in the linear stability analysis. The material is rate independent, Eq. (4) with $\rho=7800 \mathrm{~kg} / \mathrm{m}^{3}$ and $\sigma_{0}=500 \mathrm{MPa}$. Within the range $V_{0}<900 \mathrm{~m} / \mathrm{s}$ the difference between the three approaches considered arises. The fragmentation can be approached by statistical analyses such as the classical theory of Mott (1947). On contrary, within the range $V_{0}>900 \mathrm{~m} / \mathrm{s}$ the results obtained from the three approaches converge in the prediction of a critical wavelength $\left(L_{0} / \phi_{0}\right)^{\text {critical }} \approx 1.5$ which determines the spacing between necks $($ and the fragment size) when inertia is dominant in the loading process.

Identical representation, $L_{0} / \phi_{0}$ vs $V_{0}$ and $\dot{\varepsilon}_{0}$ is conducted in Figs. 19 and 20. In these cases the material properties have been varied. Fig. 19 concerns to the rate dependent material Eq. (4) with $\rho=7800 \mathrm{~kg} / \mathrm{m}^{3}, \sigma_{0}=500 \mathrm{MPa}, m=0.01$ and $\dot{\varepsilon}_{r}=1000 \mathrm{~s}^{-1}$. Fig. 19 concerns the rate dependent material Eq. (4) with $\rho=17300 \mathrm{~kg} / \mathrm{m}^{3}, \sigma_{0}=500 \mathrm{MPa}, m=0.01$ and $\dot{\varepsilon}_{r}=1000 \mathrm{~s}^{-1}$. For both material configurations the three approaches predict a value for the critical wavelength $\left(L_{0} / \phi_{0}\right)^{\text {critical }} \approx 1.5$. The difference seems to reside uniquely on the range of loading velocities for which this critical wavelength applies.

\section{Conclusions}

In this paper, the analysis of ductile rings expanding at very high strain rates has been addressed using three different methodologies: the 3D numerical simulations of the full problem, the analysis of a unitary periodic cell with loading and boundary conditions consistent with the expanding ring and, finally, by a linear perturbation method.

The main conclusions are summarized as follows:

- Fully 3D numerical simulations: It has been reported that beyond certain loading velocity all the necks nucleated develop until failure. Furthermore, the number of necks and the number of fragments saturate to the same limiting value at high strain rates. Then, the mean fragment size corresponding to saturation obeys to the ratio between fragments length $L_{0}$ (measured in the undeformed reference configuration) and ring thickness $e_{0}$ by the relation $\left(L_{0} / e_{0}\right)^{\text {critical }} \approx 1.5$. This value shows little dependence on the material description as well as on the dimensions of the sample cross section.

- Unitary periodic cell: It has been reported that combination of the stabilizing aspects of multidimensional stress effects on short cell lengths and the stabilizing aspects of inertia on long cell lengths lead to the promotion of an intermediate cell length which determines the minimum necking strain. In coincidence with the results derived from the 3D numerical computations, this minimum necking strain is dictated by the relation $\left(L_{0} / \phi_{0}\right)^{\text {critical }} \approx 1.5$ where now $L_{0}$ is defined by the imperfection wavelength (cell-size) and $\phi_{0}$ is the diameter of the circular cross section of the ring.

- Stability analysis: The analysis showed that at sufficiently high strain rates the selection of a dominant wavelength which tends to $\left(L_{0} / \phi_{0}\right)^{\text {critical }} \approx 1.5$ occurs. This value finds strong correlation with the observations derived from the $3 \mathrm{D}$ ring expansion simulations and the finite element modelling of the unitary periodic cell.

Thus, the results derived from the three methodologies addressed allow for identification of a critical wavelength which dictates the fragmentation mechanisms of ductile rings expanded at very high strain rates. This critical wavelength is revealed quite independent of the material constitutive model but closely related to material inertia by the ratio $\left(L_{0} / \phi_{0}\right)^{\text {critical }} \approx 1.5$. The analysis provides a new interpretation of the mechanisms which govern the fragmentation of ductile rings subjected to very high loading velocities.

\section{Acknowledgments}

The researchers of the University Carlos III of Madrid are indebted to the Comunidad Autónoma de Madrid (Project CCG10-UC3M/DPI-5596) and to the Ministerio de Ciencia e Innovación de España (Project DPI/2011-24068) for the financial support received which allowed conducting part of this work.

The researchers of the University Carlos III of Madrid kindly acknowledge the technical support provided by Mr. M. Moriche.

Alain Molinari acknowledges the support of the University Carlos III of Madrid with a Cátedra de Excelencia funded by Banco Santander.

\section{References}

ABAQUS/Explicit, 2010. Abaqus Explicit v6.10 User's Manual, Version 6.10 Edition. ABAQUS Inc., Richmond, USA.

Altynova, M., Hu, X., Daehn, G.S., 1996. Increased ductility in high velocity electromagnetic ring expansion. Metall. Trans. A 27, 1837-1844.

Becker, R., 2002. Ring fragmentation predictions using the Gurson model with material stability conditions as failure criterion. Int. J. Solids Struct. 39, 3555-3580.

Bridgman, P.W., 1952. Studies in large plastic flow and fracture, with special emphasis on the effects of hydrostatic pressure, vol. 1, McGraw-Hill Book Company Inc, New York, pp. 9-37.

Clark, D.S., Wood, D.S., 1950. The influence of specimen dimension and shape on the results in tension impact testing. Trans. Am. Soc. Mech. Eng. 5, 577-585. 
Fressengeas, C., Molinari, A., 1985. Inertia and thermal effects on the localization of plastic flow. Acta Metall. 33, 387-396.

Fressengeas, C., Molinari, A., 1994. Fragmentation of rapidly stretching sheets. Eur. J. Mech. A/Solids 13, 251-268.

Fyfe, I.M., Rajendran, A.M., 1980. Dynamic pre-strain and inertia effects on the fracture of metals. J. Mech. Phys. Solids $28,17-26$.

Gosh, A.K., 1977. Tensile instability and necking in materials with strain hardening and strain-hardening rate. Acta Metall. 25, 1423-1424.

Gourdin, H., 1989. Analysis and assessment of electromagnetic ring expansion as a high-strain-rate test. J. Appl. Phys. 65, 411-422.

Grady, D., 1981. Fragmentation of solids under impulsive stress loading. J. Geophys. Res. 86, 1047-1054.

Grady, D.E., 1982. Local inertia effects in dynamic fragmentation. J. Appl. Phys. 68, 322-325.

Grady, D.E., Brenson, D.A., 1983. Fragmentation of metal rings by electromagnetic loading. Exp. Mech. 12, 393-400.

Grady, D.E., Kipp, M.E., Benson, D.A., 1984. Energy and statistical effects in the dynamic fragmentation of metal rings. In: Harding, J. (Ed.), Mechanical Properties at High Strain Rates. Institute of Physics, Bristol, pp. 315-320.

Grady, D.E., Olsen, M.L., 2003. A statistics and energy based theory of dynamic fragmentation. Int. J. Impact Eng. 29, $293-306$.

Guduru, P.R., Freund, L.B., 2002. The dynamics of multiple neck formation and fragmentation in high rate extension of ductile materials. Int. J. Solids Struct. 39, 5615-5632.

Han, J.B., Tvergaard, V., 1995. Effect of inertia on the necking behaviour of ring specimens under rapid axial expansion. Eur. J. Mech. A/Solids 14, $287-307$.

Hild, F., Denoual, C., Forquin, P., Brajer, X., 2003. On the probabilistic-deterministic transition involved in a fragmentation process of brittle materials. Comput. Struct. 81, 1241-1253.

Hill, R., Hutchinson, J.W., 1975. Bifurcation phenomena in the plane tension test. J. Mech. Phys. Solids 23, 239-264.

Hu, X., Daehn, G.S., 1996. Effect of velocity on flow localization in tension. Acta Mater. 44, 1021-1033.

Hutchinson, J.W., Neale, K.W., 1977. Influence of strain rate sensitivity on necking under uniaxial tension. Acta Metall. 25, 839-846.

Janiszewski, J., 2012. Ductility of selected metals under electromagnetic ring test loading conditions. Int. J. Solids Struct. 49, 1001-1008.

Janiszewski, J., Pichola, W., 2009. Development of electromagnetic ring expansion apparatus for high-strain-rate test. Solid State Phenom., 645-650.

Juanicotena, A., Llorca, F., 1997. Utility of the ring expansion test in the analysis of different laws of elastoplastic behavior at high strain rates-application to copper and mars-190 steel. J. Phys. IV 7, 541-546.

Kàrman, T.V., Duwez, P., 1950. The propagation of plastic deformation in solids. J. Appl. Phys. $21,987$.

Kipp, M., Grady, D., 1985. Dynamic fracture growth and interaction in one dimension. J. Mech. Phys. Solids 33, 399-415.

Knoche, P., Needleman, A., 1993. The effect of size on the ductility of dynamically loaded tensile bars. Eur. J. Mech. A/Solids 12 , 586-601.

Lovinger, Z., Rikanati, A., Rosenberg, Z., Rittel, D., 2011. Electro-magnetic collapse of thick-walled cylinders to investigate spontaneous shear localization. Int. J. Impact Eng. 38, 918-929.

Mercier, S., Granier, N., Molinari, A., Llorca, F., Buy, F., 2010. Multiple necking during the dynamic expansion of hemispherical metallic shells, from experiments to modelling. J. Mech. Phys. Solids 58, 955-982.

Mercier, S., Molinari, A., 2003. Predictions of bifurcations and instabilities during dynamic extensions. Int. J. Solids Struct. 40, $1995-2016$.

Mercier, S., Molinari, A., 2004. Analysis of multiple necking in rings under rapid radial expansion. Int. J. Impact Eng. 4, 403-419.

Mott, N.F., 1947. Fragmentation of shell cases. In: Series A Proceedings of the Royal Society, London, pp. 300-308.

Needleman, A., 1991. The effect of material inertia on neck development. In: Yang, W.H. (Ed.), Topics in Plasticity. AM Press, Ann Arbor, MI, pp. 151-160.

Niordson, F.L., 1965. A unit for testing materials at high strain rates. Exp. Mech. 5, 29-32.

Pandolfi, A., Krysl, P., Ortiz, M., 1999. Finite element simulation of ring expansion and fragmentation: the capturing of length and time scales through cohesive models of fracture. Int. J. Fract. 95, 297.

Rajendran, A.M., Fyfe, I.M., 1982. Inertia effects on the ductile failure of thin rings. J. Appl. Mech. 49, 31-36.

Rodríguez-Martínez, J.A., Vadillo, G., Zaera, R., Fernández-Sáez, J., 2013. On the complete extinction of selected imperfection wavelengths in dynamically expanded ductile rings. Mech. Mater. 60, 107-120.

Rusinek, A., Zaera, R., 2007. Finite element simulation of steel ring fragmentation under radial expansion. Int. J. Impact Eng. 34, 799-822.

Shenoy, V.B., Freund, L.B., 1999. Necking bifurcations during high strain rate extension. J. Mech. Phys. Solids 47, $2209-2233$.

Sørensen, N.J., Freund, L.B., 1998. Dynamic bifurcation during high-rate planar extension of a thin rectangular block. Eur. J. Mech. A/Solids 17, 709-724.

Sørensen, N.J., Freund, L.B., 2000. Unstable neck formation in a ductile ring subjected to impulsive radial loading. Int. J. Solids Struct. 37, $2265-2283$.

Vadillo, G., Rodríguez-Martínez, J.A., Fernández-Sáez, J., 2012. On the interplay between strain rate and strain rate sensitivity on flow localization in the dynamic expansion of ductile rings. Int. J. Solids Struct. 49, 481-491.

Vela, C., Rodríguez-Martínez, J.A., Rusinek, A., 2011. FE analysis on the formation of plastic instabilities in dynamically expanded copper rings. Eng. Trans. $59,1-23$.

Walsh, J.M., 1984. Plastic instability and particulation in stretching metals jets. J. Appl. Phys. 56, 1997-2006.

Xue, Z., Vaziri, A., Hutchinson, J.W., 2008. Material aspects of dynamic neck retardation. J. Mech. Phys. Solids 56, 93-113.

Zaera, R., Fernández-Sáez, J., 2006. An implicit consistent algorithm for the integration of thermoviscoplastic constitutive equations in adiabatic conditions and finite deformations. Int. J. Solids Struct. 43, 1594-1612.

Zhang, H., Ravi-Chandar, K., 2006. On the dynamics of necking and fragmentation I. real-time and post-mortem observations in Al 6061-O. Int. J. Fract. $142,183-217$.

Zhang, H., Ravi-Chandar, K., 2008. On the dynamics of necking and fragmentation - II. effect of material properties geometrical constraints and absolute size. Int. J. Fract. 150, 3-36

Zhou, F., Molinari, J.F., Ramesh, K.T., 2005. A cohesive model based fragmentation analysis: effects of strain rate and initial defects distribution. Int. J. Solids Struct. 42, 5181-5207.

Zhou, F., Molinari, J.F., Ramesh, K.T., 2006a. Analysis of the brittle fragmentation of an expanding ring. Comput. Mater. Sci. 37 , 74-85.

Zhou, F., Molinari, J.F., Ramesh, K.T., 2006b. Characteristic fragment size distributions in dynamic fragmentation. Appl. Phys. Lett. 88, 261918.

Zhou, F., Molinari, J.F., Ramesh, K.T., 2006c. An elasto-visco-plastic analysis of ductile expanding ring. Int. J. Impact Eng. 33, 880-891. 